DOI: $10.3901 / J M E .2020 .19 .221$

\title{
陶瓷光固化技术及其应用*
}

\author{
吴甲民 $1,2,3$ 杨源祺 ${ }^{1,2}$ 王 操 ${ }^{1,2}$ 何逸宁 ${ }^{1,2}$ 石 婷 ${ }^{1,2}$ 甘 恬 ${ }^{1,2}$ \\ 陈 双 ${ }^{1,2,3}$ 史玉升 ${ }^{1,2,3}$ 王卫 4
}

(1. 华中科技大学材料科学与工程学院 武汉 430074;

2. 华中科技大学材料成形与模具技术国家重点实验室 武汉 430074;

3.增材制造陶瓷材料教育部工程研究中心 武汉 430074;

4. 浙江华科三维科技有限公司 温州 325000)

摘要: 陶瓷材料因具有高硬度、高强度、耐高温、抗腐蚀等优异性能, 被广泛应用于航空航天、生物医疗等领域, 但是应用于上 述领域的复杂结构陶瓷的制造仍然是一个重要的挑战。增材制造技术通过逐层堆积材料的方式来获得实体，在制造复杂结构陶瓷 方面具有传统成形方式所无法比拟的优势。在众多陶瓷增材制造技术中，陶瓷光固化技术因其理想的成形质量而受到广泛关注和 重视。在介绍立体光固化、数字光处理这两种主流陶瓷光固化技术的原理和特点的基础上，系统地介绍和分析了立体光固化技术 和数字光处理技术的研究现状、应用以及存在的问题。最后，对陶瓷光固化技术及其应用进行了总结和展望。

关键词：陶瓷；增材制造；光固化技术；立体光固化；数字光处理

中图分类号: TQ174

\section{Photopolymerization Technologies for Ceramics and Their Applications}

\author{
WU Jiamin $^{1,2,3}$ YANG Yuanqi ${ }^{1,2}$ WANG Cao ${ }^{1,2}$ HE Yining $^{1,2}$ SHI Ting ${ }^{1,2}$ GAN Tian ${ }^{1,2}$ \\ CHEN Shuang $^{1,2,3}$ SHI Yusheng ${ }^{1,2,3}$ WANG Wei ${ }^{4}$
}

(1. School of Materials Science and Engineering, Huazhong University of Science and Technology, Wuhan 430074;

2. State Key Laboratory of Materials Processing and Die \& Mould Technology, Huazhong University of Science and Technology, Wuhan 430074;

3. Engineering Research Center of Ceramic Materials for Additive Manufacturing, Ministry of Education, Wuhan 430074;

4. Zhejiang Huake 3D Technology Co., Ltd., Wenzhou 325000)

\begin{abstract}
Because of the excellent properties in hardness, strength, temperature resistance and corrosion resistance, etc., ceramic materials are widely used in various fields such as aerospace, biomedical and so on. However, it is still a vital challenge for the manufacturing of ceramics with complex structures applied in the above fields. Additive manufacturing (AM) technology obtains objects by stacking materials layer by layer, which has significant advantages compared with traditional forming methods in manufacturing complex ceramics. Among numerous ceramic AM technologies, ceramic photopolymerization technologies have received extensive attention due to the ideal fabrication quality. Based on the introduction of the principles and characteristics of two mainstream ceramic photopolymerization technologies: stereolithography (SL) and digital light processing (DLP), their research status, applications and existing problems are introduced and analyzed. In the end, a summary and prospect of the ceramic photopolymerization technologies are put forward.
\end{abstract}

Key words: ceramics; additive manufacturing; photopolymerization technologies; stereolithography; digital light processing

* 国家自然科学基金(51975230)、国家重点研发计划 (2018YFB1105503)、湖北 省技术创新专项重大项目(2019AAA002)、高性能陶瓷和超微结构国家 重点实验室开放课题(SKL201903SIC)和国家级大学生创新创业训练 计划(202010487001)资助项目。20200428 收到初稿, 20200528 收到修改稿 


\section{0 前言}

作为三大固体材料之一，陶瓷材料因具有高硬 度、高强度、耐高温、抗腐蚀、良好的绝缘性、生 物相容性等优异的性能, 被广泛应用于航空航天、 生物医疗等领域 ${ }^{[1-3]}$ 。随着科学技术的不断进步和工 业生产需求的不断发展, 诸如航空发动机浴轮叶片 铸造用陶瓷型芯、人工骨支架等高性能复杂结构陶 瓷零件的高精度、可重复性制造越来越受到人们的 关注 ${ }^{[4-6]}$ 。陶瓷材料具有的高硬度和脆性使得对其进 行机加工存在很大的困难。目前, 人们也采用注浆 成型、凝胶注模成型、直接凝固注模成型等工艺制 造复杂结构陶瓷 ${ }^{[7-9]}$, 但是, 这些工艺存在依赖模具 等问题, 且部分特别复杂的陶瓷甚至无法制造, 因 而难以满足高性能陶瓷产品的快速开发和广泛应用 的要求 ${ }^{[10-11]}$ 。

增材制造(Additive manufacturing, AM)技术自 20 世纪 80 年代提出以来就引起了人们的广泛关注, 其基本原理为将三维模型切片、再通过逐层累积的 方式来获得实体，相较于传统的减材制造，增材制 造具有材料利用率高、成形难度不受模型复杂程度 的影响等优点，已被逐渐应用于高性能复杂陶瓷零 件的个性化设计和制造 ${ }^{[2-16]}$ 。目前主要的增材制造 技术包括熔融沉积成形(Fused deposition modeling, FDM)、激光选区烧结(Selective laser sintering, SLS)、 激光选区熔化(Selective laser melting, SLM)、分层 实体制造(Laminated object manufacturing, LOM)、 光固化等 ${ }^{[17-19]}$ 。

在众多陶瓷材料的增材制造技术中，陶瓷光固 化技术因具有理想的成形质量而受到广泛关注和重 视。陶瓷光固化技术采用光敏陶瓷浆料或膏体为原 料, 利用特定波长的光源控制其逐层固化, 根据曝 光图案形成原理和控制系统的不同, 其主要可分为 以下三种：立体光固化(Stereolithography, SL)技 术、数字光处理(Digital light processing, DLP)技 术和双光子聚合(Two-photon polymerization, TPP) 技术 ${ }^{[20-21]}$, 其中, TPP 技术具有优异的空间选择性, 在高分子材料的微纳尺度制造领域具有较高的应用 价值 ${ }^{[22-23]}$, 但对陶瓷浆料的透光性能要求较高, 限制了其在陶瓷增材制造领域的应用。本文详细 阐述了立体光固化和数字光处理这两种陶瓷光固 化技术的原理、发展历程、研究现状及其应用, 在此基础上分析了陶瓷光固化技术目前存在的问 题并指出其发展趋势。

\section{1 陶瓷光固化技术简介}

\section{1 立体光固化技术简介}

SL 技术最早由美国人 HULL 于 1986 年提出

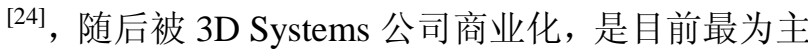
流的增材制造技术之一。陶瓷 SL 技术的工作原理 如图 1 所示, 紫外激光束聚焦到浆料表面形成光 斑, 光斑在扫描振镜的作用下根据导入的切片文件 信息在浆料表面进行单层图案的轮廓和填充扫描, 光斑经过处陶瓷浆料迅速固化, 而光斑未经过处浆 料仍为液态, 底层图案扫描完成后, 打印平台下降 一个层厚的高度, 通过刮刀的运动涂覆一层新的陶 瓷浆料, 然后进行下一层图案的扫描, 陶瓷浆料固 化后线与线、层与层之间紧密粘接。重复以上步骤, 最终获得组分均匀的陶瓷素坏。

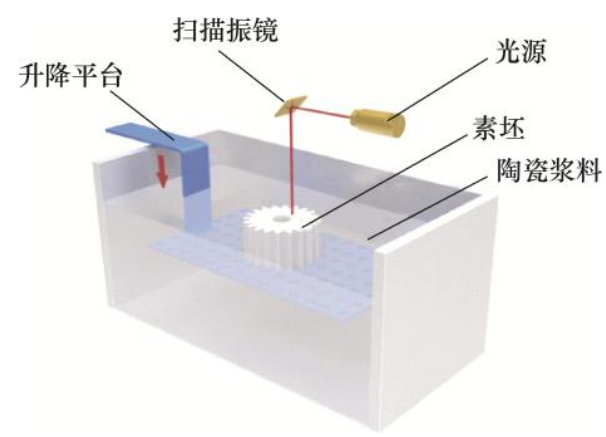

图 1 立体光固化技术原理图 ${ }^{[1]}$

SL 技术最初用于液态光敏树脂的成形, 由于其 利用光斑进行逐点扫描, 因此可以获得较高的精度, 但同时, 光斑并非理想的点, 因而在加工微小结构 及轮廓时需要考虑光斑实际形状和尺寸的影响, 为 了解决这一问题从而提高 SL 技术的成形速度和成 形精度, 国内外研究者提出了许多优化的工艺方案。 其中, 一个较为重要的思路是根据扫描区域的特征 改变光斑尺寸，并优化其扫描路径。潘翔等 ${ }^{[25]}$ 提出 了一种分区域变补偿量的光斑扫描方法, 有效地提 高了 SL 制件中微小结构的成形精度。李子健 ${ }^{[26]}$ 进 行了变光斑工艺及其配套的路径规划研究, 提出了 一种连续可变光斑扫描方法, 在提高精度的同时避 免了局部区域过固化现象，并且提高了成形效率。 另外, 涂层的涂覆质量直接决定了垂直方向的分辨 率，因此，降低最小涂层厚度也是提高成形分辨率 的途径之一。胥光申等 ${ }^{[27-28]}$ 围绕高分辨率光固化设 备进行了光学系统、涂层系统等的搭建, 提出了多 点定量精密补充树脂的涂层方法, 研发出的 SL 设 备最小涂层厚度低至 $0.02 \mathrm{~mm}$, 并利用该设备制备 
出了 $0.011 \mathrm{~mm}$ 壁厚的单壁结构和 $0.034 \mathrm{~mm}$ 宽的 槽状结构, 实现了光敏树脂的较高精度的打印。 上世纪 90 年代, GRIFFTH 等 ${ }^{[29]}$ 使用可以发生光 聚合反应的高浓度陶瓷浆料代替液态光敏树脂, 并从实验上验证了 Beer-Lambert 定律对陶瓷浆料 光固化的适用性, 进而提出了陶瓷浆料光固化深 度的修正公式, 为陶瓷光固化技术的研究和应用 奠定了理论基础, 开拓了 SL 技术在陶瓷增材制造 领域中的应用。

高性能陶瓷浆料的制备是陶瓷 SL 技术得以应 用和获得高性能陶瓷零件的关键环节。经过长期 的探索, 光固化陶瓷浆料的配方已经形成了相对 固定的体系, 其主要包含陶瓷粉体、光敏树脂、 光引发剂、分散剂等成分。目前, 根据分散介质 的不同, 光固化陶瓷浆料主要可以分为树脂基和 水基两种体系, 国内外研究者围绕这两种体系的 陶瓷浆料都开展了许多工作。

树脂基光固化陶瓷浆料通常采用丙烯酸酯等作 为分散介质, 光引发剂吸收特定波长的紫外光后引 起光敏树脂迅速交联并固化, 形成包含有陶瓷颗粒 的固态聚合物网状基质, 从而使素坏具备一定的强 度 ${ }^{[30]}$ 。DING 等 ${ }^{[31]}$ 系统地研究了反应单体、分散剂、 粉体粒径、固含量和球磨时间对 $\mathrm{SiC}$ 陶瓷浆料的分 散性、流变性和稳定性的影响, 并综合考虑了浆料 的黏度和固化效果之后, 选取质量比为 1:1 的 1,6己二醇二丙烯酸酯(HDDA)和三着圣甲基丙烷三丙烯 酸酯(TMPTA) 树脂混合物作为反应单体, 实验结果 表明, 向微米级 $\mathrm{SiC}$ 颗粒中混入 $1 \%$ (质量分数)的纳 米 $\mathrm{SiC}$ 颗粒能够有效提高浆料的稳定性, 同时还发 现, 随着球磨时间的增加, 浆料的黏度也会随之增 大。SUN 等 ${ }^{[32]}$ 使用丙烯酰吗啉(ACMO)和聚乙二醇 二丙烯酸酯(PEGDA)的混合物作为分散介质配制纳 米 $\mathrm{ZrO}_{2}$ 浆料, 并对比了硬脂酸 $(\mathrm{SA})$ 、油酸 $(\mathrm{OA}) 、$ BYK、KH560、variquat CC 五种分散剂对浆料性能 的影响, 发现加入 BYK 的浆料稳定性和流动性均 为最优。这是因为 $B Y K$ 的端链具有亲油性, 同时 其磷酸酯基团针固在 $\mathrm{ZrO}_{2}$ 颗粒表面, 从而形成了稳 定的空间位阻效应。

水基光固化陶瓷浆料的固化原理与树脂基陶 瓷浆料相同, 但其用水性低聚物代替光敏树脂中的 低聚物或者用水稀释一定量的低聚物或反应单体, 而水的黏度低于各类光敏树脂, 且容易通过干燥从 成形样品中去除, 因此, 水基陶瓷浆料具有黏度低、 有机物含量少、污染小等优点 ${ }^{[33]}$ 。宁会峰等 ${ }^{[34]}$ 开 展了水基光固化陶瓷浆料的性能研究, 并通过硅烷
偶联剂 $\mathrm{KH} 570$ 对 $3 \mathrm{Y}^{-} \mathrm{ZrO}_{2}$ 纳米粉改性获得了分散 良好的水基浆料。单线固化实验结果表明, 和液态 光敏树脂相比较, 水基陶瓷浆料的单条固化线横截 面更宽且更浅。WANG 等 ${ }^{[35]}$ 首先将反应单体丙烯 酰胺和交联剂 N-N'亚甲基双丙烯酰胺加入去离子 水中并搅拌溶解, 随后加入一定量的经聚丙烯酸 钠改性的羟基磷灰石(HAP)粉体和甘油, 最后加 入光引发剂 1173 , 制备出了固含量为 $52 \%$ (体积 分数)的水基 HAP 陶瓷浆料, 其临界曝光量和透 射深度分别为 $20.3 \mathrm{~mJ} / \mathrm{cm}^{2}$ 和 $50.7 \mu \mathrm{m}$ 。由于水基 陶瓷浆料所得的光固化素坏强度较低, 且有关其 排胶、烧结工艺的研究较少，限制了其广泛应用。 目前, 树脂基光固化陶瓷浆料的研究和应用仍占 主导地位 ${ }^{[36-37]}$ 。

浆料的固化性能也是陶瓷的光固化制备流程 中需要考虑的重要因素。向液态光敏树脂中加入 陶瓷粉体后, 陶瓷颗粒对紫外光的吸收和散射作 用会对光敏树脂的光聚合反应产生影响, 致使陶 瓷浆料的固化深度降低, 进而可能影响陶瓷素坏 的层间粘接强度, 最终影响陶瓷零件的性能。针 对这一问题, 国内外围绕陶瓷浆料光固化的影响 因素开展了一系列理论和实验研究。SUN 等 ${ }^{[38]}$ 通 过建立数值模型研究了不同参数对 $\mathrm{SiO}_{2} 、 \mathrm{Al}_{2} \mathrm{O}_{3}$ 、 锆钛酸铅 $(\mathrm{PZT})$ 三种陶瓷浆料的光固化效果的影 响, 发现当陶瓷粉体的粒径与紫外光波长相差越 小、陶瓷材料与光敏树脂的折射率相差越大时, 散射作用越严重、固化深度越小。此外, 光敏树 脂配方对浆料的光聚合动力学也有较大影响。

BADEV 等 ${ }^{[39]}$ 通过实验发现, 向聚醚丙烯酸酯 (PEAAM)中加入活性稀释剂 HDDA, 一方面降低 了浆料黏度、增加了反应官能团的迁移率从而提 高转化率, 另一方面降低了官能团密度而不利于 聚合产率的提高, 同时指出晶间相的黏度是影响 光聚合动力学的唯一参数, 而陶瓷浆料的整体流 变性能不会影响光聚合过程中反应官能团的扩 散, 并且得到了和 SUN 等 ${ }^{[38]}$ 一致的结论。但 CHARTIER 等 ${ }^{[40]}$ 指出, 陶瓷浆料流变性能对光聚 合动力学的影响只在浆料固含量较低时可以被忽 略, 而当浆料固含量足够高时, 则必须考虑陶瓷 颗粒之间以及陶瓷颗粒与反应单体之间的相互作 用, 这些相互作用会降低光敏树脂的转化率和聚 合速率。LIU 等 ${ }^{[41]}$ 通过 KH560 对 $\mathrm{Si}_{3} \mathrm{~N}_{4}$ 粉体进行 表面改性, KH560 的环氧基与环氧丙烯酸酯(EA) 的羟基形成醚键，从而促使 $\mathrm{EA}$ 在 $\mathrm{Si}_{3} \mathrm{~N}_{4}$ 颗粒表面 形成壳层结构。由于 $\mathrm{EA}$ 壳层的存在, 粉体与树脂 
的折射率之差大大减小, 从而增加了浆料的固化 深度。

此外, 也有少部分研究使用黏度较高的光敏陶 瓷膏体作为 SL 的打印原料。LIU 等 ${ }^{[42]}$ 使用质量比 为 3:7 的双三羟甲基丙烷丙烯酸酯(Di-TMPTA)和 HDDA 的混合树脂作为反应单体, 制备了固含量高 达 $46.8 \%$ (体积分数)的 $\mathrm{ZrO}_{2}$ 增韧 $\mathrm{Al}_{2} \mathrm{O}_{3}$ (ZTA) 陶瓷膏 体, 光固化素坏经烧结后密度可达 $3.79 \mathrm{~g} / \mathrm{cm}^{3}$ 。XING 等 ${ }^{[43]}$ 提出了一种新型的 $\mathrm{SiC}$ 晶须增㓞 $\mathrm{Al}_{2} \mathrm{O}_{3}$ 陶瓷膏 体的制备工艺, 其利用丁酮-乙醇的低沸点溶剂促进 $\mathrm{SiC}$ 晶须和 $\mathrm{Al}_{2} \mathrm{O}_{3}$ 粉末在树脂中的分散, 有效地防止 了晶须的团聚, 之后再通过蒸发去除溶剂, 获得了含 $15 \%$ (体积分数)的 $\mathrm{SiC}$ 晶须、固含量为 $50 \%$ (体积分数) 的 $\mathrm{SiC}_{\mathrm{w}} / \mathrm{Al}_{2} \mathrm{O}_{3}$ 膏体, 在剪切速率为 $30 \mathrm{~s}^{-1}$ 的条件下黏 度最高达 $29500 \mathrm{mPa} \cdot \mathrm{s}$, 且在实验范围内固化深度最 低为 $0.061 \mathrm{~mm}$, 性能满足打印要求。

目前, 主流的 SL 设备大多采用下沉式打印平 台, 单层陶瓷浆料的固化效果依赖于浆料的涂覆质 量, 然而, 受到机械误差以及陶瓷浆料相对较高的 黏度等因素的影响, 无论实验还是应用中都往往难 以保证每层浆料涂覆的厚度均匀且精确。另外, 浆 料涂覆的过程中刮刀的运动可能会破坏光敏聚合物 的交联密度, 降低素坏的结构完整性, 从而造成宏 观或微观上的缺陷。

由于实验和生产的需要, 国内外均涌现了一批 陶瓷 SL 设备生产商, 其中最具有代表性的是法国 3DCeram 公司, 该公司自 2001 年起就致力于陶瓷 SL 设备的研发, 并于 2015 年推出首台工业级陶瓷 光固化设备 Ceramaker 900, 打印幅面达到 $300 \times$ $300 \times 150 \mathrm{~mm}^{3}$, 同时还开发了适配的 3DMIX 系列 (包括 $\mathrm{Al}_{2} \mathrm{O}_{3} 、 \mathrm{ZrO}_{2} 、 \mathrm{TCP} 、 \mathrm{HAP}$ ) 打印膏体, 并基于 陶瓷亳体创新地提出了装配式 SL 打印工艺, 实现 了部分薄片状及细微小型陶瓷件打印过程的去支撑 化。美国 Formlabs 公司主要生产桌面级高精度陶瓷 SL 设备, 并且推出了打印件清洗和后固化设备。国 内专注于陶瓷光固化的企业较少, 陶瓷 SL 设备主 要由激光制造领域的企业研发生产。深圳光韵达公 司的 CeraBuilder 系列设备使用的是自主设计的刮 刀, 可实现浆料层厚度的精确控制。另外, 昆山博 力迈、武汉因泰莱等公司也推出了相应的陶瓷 SL 设备。

\section{2 数字光处理技术简介}

DLP 技术与 SL 技术的基本原理相同, 即通过 紫外光的选择性照射来控制陶瓷浆料的局部固化, 不同的是 DLP 是一种基于动态掩膜的光固化技术。
DLP 分上曝光和下曝光两种, 下面以应用更为广泛 的下曝光式 DLP 技术为例进行介绍。陶瓷 DLP 技 术的工作原理如图 2 所示, 树脂槽中盛有陶瓷浆 料, 成形开始后, 利用紫外光将经过数字处理的图 像自下而上地投影到成形面上, 曝光区域的陶瓷浆 料迅速固化并粘附在打印平台上, 每层图案曝光后, 打印平台上升, 陶瓷浆料依靠重力流平或通过刮刀 刮平后进行打印平台的重新定位, 然后进行下一层 的曝光。利用掩膜曝光来制造实体的方法最早由 TAKAGI 等 ${ }^{[44]}$ 于 1993 年提出, 他们使用的是成本 较高、制作工艺复杂的石英掩膜, 且分辨率远远达 不到实际要求。BERTSCH 等 ${ }^{[45]}$ 在 1997 年提出利 用 LCD 液晶显示屏生成动态掩膜, 并实现了毫米级 器件的打印, 所得制件的表面精度优于一般的扫描 式 SL 设备, 但由于液晶分子与紫外光之间的相互 作用, 该方法仍存在图像对比度低、打印件精度低、 设备使用寿命短等缺点。目前, DLP 技术的核心元 件是德州仪器(Texas Instruments, TI)公司于 1987 年 研发出的 DMD(Digital Micromirror Device)芯片, 该 元件由上百万个微镜构成的阵列组成, 其中每个微 镜对应成形面上的一个像素点, 利用反射光束定向 技术控制掩膜图像的显示输出, 从而实现高精度的 面曝光 ${ }^{[46]}$ 。

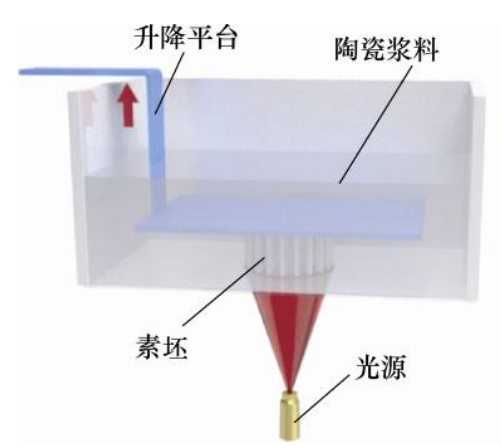

图 2 数字光处理技术原理图 ${ }^{[1]}$

光源和投影系统相对成熟后, 在 DLP 技术的 基础之上, 为了提高打印速度, 陆续出现了一些 改进的面曝光增材制造工艺。TUMBLESTON 等 ${ }^{[47]}$ 于 2015 年提出一种与 DLP 技术相类似的连续液 面制造(Continuous liquid interface production, CLIP)技术, 其利用一层高透光、高透氧膜作为离 型膜, 由于光敏树脂的氧阻聚效应, 底层树脂的 光固化受到抑制因而始终保持液态, 从而使固化 层始终与离型膜分离、保证了曝光过程的连续性, 这也使得打印件表面不会产生其他增材制造技术 不可避免的台阶效应, 很好地解决了打印件力学 性能各向异性的问题, 同时避免了固化层通过拉 
力与离型膜分离时产生缺陷。但是, 液态死区的 厚度常常只有数微米, 相较于流动性好的液态光 敏树脂, 高黏度的陶瓷浆料难以填充其中, 另外, 为了保证液体的快速、连续填充, CLIP 技术对打 印模型的结构有一定要求, 一般需要将模型进行 镂空设计, 因而不具有普适性。2019 年, KELLY 等 ${ }^{[48]}$ 又提出了计算机轴向光刻(Computer axial lithography, CAL)技术, 与普通的面曝光成形方式 截然不同, 他们采用 DLP 投影设备将连续的图像 投影至绕轴旋转的树脂内部, 零件直接在回转轴 处成形, 零件的成形速度和表面质量都得到了显 著提高, 并且, 这种技术对材料的流动性没有要 求, 高黏度的树脂反而有利于打印过程的去支撑 化, 但其精度只能控制在毫米级, 有待进一步研 究改进。虽然上述两种技术在原理上做出了创造 性的改进, 但其在陶瓷零件制造上的应用价值尚 未得到体现。目前, 陶瓷的面曝光增材制造的研 究和应用仍以 DLP 技术为主。

采用 DMD 面曝光使得 DLP 技术的成形精度 可达数微米, 但同时也衍生出了新的问题。根据 DLP 技术的工作原理可知, 投影面上各点到对应 微镜的距离不同, 故投影图像上各处的曝光能量 并非是均匀的, 而是由中心向四周逐渐递减, 这 就使得不同位置的陶瓷浆料固化深度存在一定差 异, 从而引起固化层产生内应力, 容易导致后处 理过程中开裂等严重缺陷的产生, 甚至因为翅曲 变形造成打印失败, 因此, 优化和改进现有技术 和工艺方案从而克服可能产生的缺陷显得尤为重 要。首要的解决方法是实现曝光过程中光照强度 的均匀化, 其中, 较为普遍的一种思路是控制投 影图像的灰度。邱志惠等 ${ }^{[49]}$ 在 DLP 设备的上位软 件中添加灰度补偿掩膜, 与原始曝光掩膜相互叠 加后可获得较为均匀的光强分布, 但这种对全平 面的光照强度调整方法降低了光照利用率, 从而 延长了打印时间, 并且对于不同型号的设备可移 植性较差。基于此, 冊立芳等 ${ }^{[50]}$ 提出了一种模型 自适应光照均匀化方法, 首先针对不同的模型切 片进行最大曝光区域的划定, 然后计算该区域内 的最优灰度分布, 光敏树脂的固化实验结果表明, 该方法相较于全平面灰度调整在光照利用率上具 有明显的优越性。上述对于图像灰度的控制方法 需要采集较多的样本点, 从而增加了实际操作的 复杂度, 同时还需要较为精密的检测仪器, 因此, 陈国大 等 ${ }^{[51]}$ 提出了一种简便的光照补偿方法, 其采用投影图像从中心向四周逐渐消失的方式代
替瞬间消失，从而使光强较小处获得更长时间的 曝光, 进而实现补偿曝光能量的效果。除了上述 对曝光掩膜的改进方法外, 优化浆料配方以及改 进清洗干燥、排胶烧结等后处理工艺也是抑制缺 陷产生的有效途径。EMIL 等 ${ }^{[52]}$ 研究了 $\mathrm{Al}_{2} \mathrm{O}_{3}$ 陶瓷 浆料中树脂组分对陶瓷烧结体缺陷的影响, 发现 引入不参与光聚合反应的组分可以减小素坏的收 缩率从而降低内应力, 同时还能改善排胶过程从 而减少层间和层内裂纹的产生。

另外, 提高 DLP 技术的最大成形尺寸也是目 前亟需攻克的难题。虽然 DMD 芯片具有较高的分 辨率, 但由于其物理分辨率相对固定, 在制造大 尺寸零件时容易出现图像失真的问题, 使得 DLP 设备的成形台面尺寸十分受限。WU 等 ${ }^{[53]}$ 将三角 洲结构用于 DLP 面曝光增材制造设备, 提高了打 印平台的自由度, 使其能够在水平面内运动, 并 结合新型的图形分析算法, 通过拼接式的曝光实 现了大尺寸零件的打印, 这种方法的主要问题在 于难以有效地控制机械系统的误差, 且打印时间 随着曝光面积成倍地增加。赵立东等 ${ }^{[54]}$ 摒弃了原 有的移动打印平台的思路, 而采用多光源组合进 行同时曝光, 较为全面地考虑了曝光重叠区域的 过固化以及光照不均匀的问题, 并通过模型分析、 光照度调节等手段提高了打印件的质量, 但增加 光源必定带来成本的大幅上升。权坤 ${ }^{[5]}$ 基于紫外 光下臭氧和氧气可发生相互转化的原理, 提出了 一种双紫外臭氧掩膜面成形方案, 其在密闭的氧 气环境下, 先利用紫外光将局部氧气转化为臭氧, 再利用另一种波长的紫外光进行大面积均匀曝 光, 此时该波长的紫外光可以透过氧气区引发树 脂固化, 而臭氧则会吸收紫外光并迅速转化为氧 气, 致使该区域的树脂无法固化。这一方案中 DMD 芯片仅被用于生成小面积的非曝光掩模, 而大面 积的均匀曝光可由其他非 DMD 光路系统完成, 有 利于提升 DLP 设备的成形尺寸。

总体而言, 采用自下而上的投影方式的 DLP 技 术, 实际固化层厚不受浆料涂覆效果的影响, 因此 相较于 SL 技术在坚直方向上具有更高的成形精 度 ${ }^{[56]}$, 同时具有节省原料的优点。但是, 打印过程 中固化层与离型膜的分离拉力可能导致素坏损坏, 另外, 光照强度的不均匀性不能完全消除, 其造成 的内应力尽管对于单层的影响可以降至较低水平, 但逐层累加后的效果仍较为显著, 尤其是对于一些 实心或者厚壁的陶瓷零件, 素坏在清洗干燥、排胶 烧结的过程中极易开裂甚至破碎, 这也是 DLP 技术 
未来想要应用于制造大尺寸陶瓷零件必须克服的主 要问题之一。

DLP 技术及设备的研发起步较晚, 但同样也吸 引了国内外一些企业的关注。奥地利 Lithoz 公司的 Lithography-based ceramic manufacturing(LCM)陶瓷 光固化技术采用的是早期的 LED 光源, 已成功 应用于心脏起搏泵、陶瓷叶片型芯等产品的打 印。荷兰Admatec 公司于 2016 年推出其首台陶 瓷光固化设备 ADMAFLEX 130, 该设备采用簿 辊输送机构实现浆料的循环利用, 提高了原料 利用率。法国 Prodways 公司研发的基于 MOVINGLight 技术的陶瓷 DLP 设备采用了可 移动的垂直曝光光源, 在成品率和打印效率上 具有显著优势, 而且能够实现较大尺寸陶瓷零 件的制造和批量化生产。近年来, 国内的北京 十维科技、浙江迅实科技、深圳长朗智能等公 司也成功研发了一系列高性能的桌面级和工业 级陶瓷 DLP 打印机, 其中, 北京十维科技的 AUTOCERA-M 陶瓷打印机作为该公司的主流 产品, 可兼容的浆料黏度高达 $20000 \mathrm{mPa} \cdot \mathrm{s}$ 、 刮刀涂覆最小层厚低至 $25 \mu \mathrm{m}$, 已在精密铸造、 口腔医疗、文创产品等领域实现应用。

\section{2 陶瓷光固化技术的应用}

\section{1 立体光固化技术的应用}

作为最早出现的增材制造技术之一, SL 技术 在满足陶瓷结构、性能等方面要求的同时, 克服 了陶瓷在加工中带来的刀具损坏、加工精度低等 问题, 还具有设计自由度高、产品研发周期短等 优势。

目前, 报道最为广泛的主要是以 $\mathrm{Al}_{2} \mathrm{O}_{3} 、 \mathrm{ZrO}_{2}$ 等为代表的氧化物结构陶瓷。XING 等 ${ }^{[57]}$ 研究了 SL 光固化 $\mathrm{Al}_{2} \mathrm{O}_{3}$ 陶瓷素坯的烧结工艺, 通过向浆 料中加入增塑剂聚乙二醇(PEG-400), 有效改善了 素坏的层间粘接强度, 进而避免在排胶烧结过程 中层间内应力导致的坯体开裂, 并确定 $22 \%$ (质量 分数)PEG- 400 的添加可使烧结体在力学性能上 和传统干压法生产的 $\mathrm{Al}_{2} \mathrm{O}_{3}$ 陶瓷相当。 $\mathrm{ZHOU}$ 等 ${ }^{[58]}$ 在 $30 \%$ (体积分数) $\mathrm{Al}_{2} \mathrm{O}_{3}$ 的丙烯酰胺水溶液中加入 $0.4 \%$ (质量分数)的聚乙烯吡咯烷酩作为分散剂制 备浆料, 利用 SL 技术打印素坏后经真空热解和空 气排胶后获得如图 3a 所示的致密的 $\mathrm{Al}_{2} \mathrm{O}_{3}$ 陶瓷刀 具, 其相对密度高达 $99.3 \%$ 。ZHANG 等 ${ }^{[59]}$ 首次使 用微立体光固化技术(Micro-stereolithography, $\mu \mathrm{SL}$ )
成功制备微结构陶瓷，该零件尺寸与单条固化线 的宽度和深度仅相差一个数量级, 因为要尽可能 避免陶瓷颗粒的散射作用对成形精度的影响, 所 以该实验采用了黏度较低、固含量为 $33 \%$ (体积分 数) $\mathrm{Al}_{2} \mathrm{O}_{3}$ 悬浮液, 并制备出如图 $3 \mathrm{~b}$ 所示的直径为 $400 \mu \mathrm{m}$ 的 $\mathrm{Al}_{2} \mathrm{O}_{3}$ 微齿轮, 可用于微机电系统中的 高功率微传感器。 $\mathrm{HE}$ 等 ${ }^{[60]}$ 提出了一种基于 SL 的 悬浮包围投影立体光固化 (Suspension-enclosing projection stereolithography，SEPS)技术，其利用 光敏树脂 FLGPCL01、30\% 40\%(体积分数)的 $\mathrm{Al}_{2} \mathrm{O}_{3}$ 粉体、 $0.8 \%$ (质量分数)的分散剂磷酸盐酯 PS-131 制备出的陶瓷浆料, 借助固有的颗粒间阻 力来平衡固化部分的自重, 从而实现打印过程的 去支撑化。图 3c 为通过 SEPS 工艺制造的烧结后 的悬臂管结构, 证明了 SEPS 工艺在制造复杂陶瓷 悬垂构件仿生热交换器、微反应器等方面的可行 性。与之类似的, SONG 等 ${ }^{[61]}$ 也利用高黏度的浆 料作支撑从而实现 $\mathrm{ZrO}_{2}$ 零件打印过程的去支撑 化, 其使用 3-氨丙基三甲氧基硅烷(APTMS)在纳 米 $\mathrm{ZrO}_{2}$ 粉体表面形成高黏度涂层, 同时为了解决 浆料流变性能不佳的问题, 向其中加入异丙醇(IPA) 作为稀释剂, 并利用对照实验确定添加 $20 \%$ (质量 分数)的 IPA 为宜。XING 等 ${ }^{[62]}$ 研究了 ZTA 陶瓷浆 料的流变性能, 修正了反映浆料粒度分布与黏度 之间函数关系的 K-D 公式并计算出不同 $\mathrm{ZrO}_{2}$ 含量 的 ZTA 陶瓷浆料的黏度。计算和光固化实验的结 果表明, 增大 $\mathrm{ZrO}_{2}$ 的比例对改善浆料流变性能和 提高固化深度具有相反的作用, 最终制备出如图 $3 \mathrm{~d}$ 所示断裂韧性达 $7.4 \mathrm{MPa} \cdot \mathrm{m}^{1 / 2}$ 、相对密度为 $99.4 \%$ 的 ZTA 陶瓷齿轮。

另外,一些非氧化物结构陶瓷也表现出优异的 机械性能, 但由于材料折射率等因素的影响导致 浆料中紫外光散射现象严重, 浆料固化困难, 因 此利用 $\mathrm{SL}$ 技术直接打印 $\mathrm{Si}_{3} \mathrm{~N}_{4} 、 \mathrm{SiC}$ 等非氧化物陶 瓷的难度较高, 有关报道也比较少见。 BRINCKMANN 等 ${ }^{[63]}$ 开发出了一种 SiOC 基聚合 物复合材料, 利用 SL 技术打印添加有 $\mathrm{SiC}$ 晶须的 聚乙烯基三甲氧基硅烷(VMS)和聚乙二醇二丙烯 酸酯(PEGDA)树脂混合液, 该方法利用烧结过程中 $\mathrm{SiC}$ 与有机物中碳的交联生成 $\mathrm{SiOC}$, 同时通过调 节 $\mathrm{SiC}$ 晶须的添加量使零件具有理想的力学性能, 最终制备出如图 3e 所示的具有复杂形状的 SiOC 陶瓷零件。LIU 等 ${ }^{[64]}$ 利用 KH560 对 $\mathrm{Si}_{3} \mathrm{~N}_{4}$ 粉体进行 表面改性, 并加入 $1 \%$ (体积分数)的分散剂 Darvan 配制出固含量为 $45 \%$ (体积分数)的浆料, 经光固 
化、排胶、烧结得到密度为 $3.28 \mathrm{~g} / \mathrm{cm}^{3}$ 、断裂韧性为 $5.82 \mathrm{MPa} \cdot \mathrm{m}^{1 / 2}$ 的 $\mathrm{Si}_{3} \mathrm{~N}_{4}$ 陶瓷。

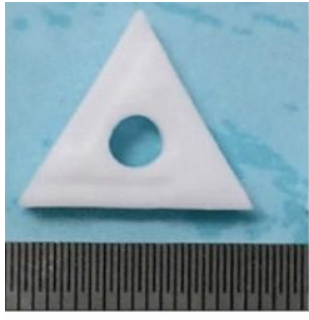

(a) $\mathrm{Al}_{2} \mathrm{O}_{3}$ 陶瓷刀具 ${ }^{[58]}$

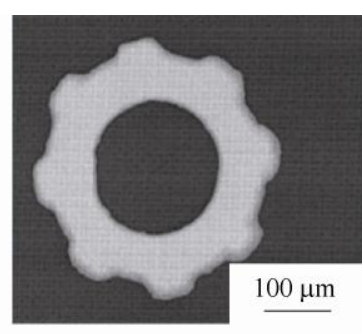

(b) $\mathrm{Al}_{2} \mathrm{O}_{3}$ 陶瓷微齿轮 ${ }^{[59]}$

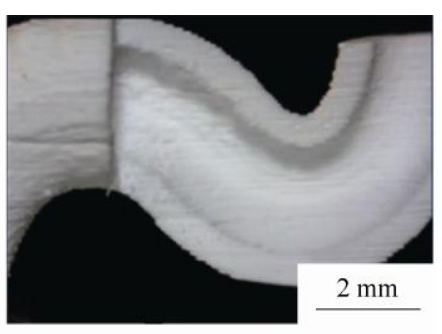

(c) 悬臂管结构 [60]

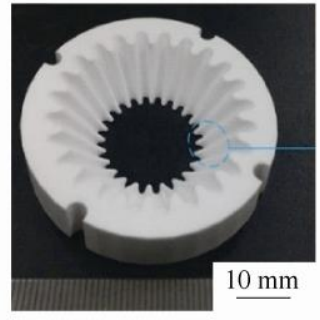

(d) ZTA 陶瓷齿轮 ${ }^{[62]}$

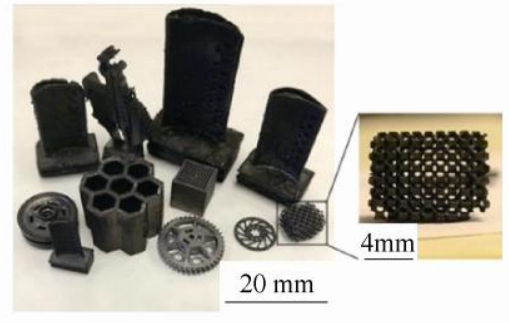

(e) SiOC 陶瓷 ${ }^{[63]}$

图 3 基于 $\mathrm{SL}$ 的结构陶瓷零件

随着传统方法越来越难以满足生物植入体个 性化设计和制造的要求, 利用 SL 技术制造生物多 孔陶瓷的优势逐渐体现。CHEN 等 ${ }^{[65]}$ 利用 SL 技术 制备 HAP 陶瓷素坏, 并研究其烧结过程中的热失 重行为, 总结出了对细胞有生物毒性的树脂材料 的热解规律。该实验设计并打印出图 4a 所示的 HAP 植入体, 在后续的动物实验中表现出良好的 生物相容性, 可用于骨组织再生工程。贲玥等 ${ }^{[66]}$ 通过加入 $2.0 \%$ (质量分数)的 $\mathrm{KH}-560$ 改善浆料的 分散性能, 并使用固含量为 $48 \%$ (质量分数)的浆料 制备出磷酸三钙(TCP)脊椎骨模型素坏。如图 $4 \mathrm{~b}$ 所示, 烧结后的陶瓷表面光滑, 气孔分布均匀, 孔隙率达 $63.9 \%$, 相对密度为 $85.8 \%$ 。MAKVANDI 等 ${ }^{[67]}$ 使用硅烷偶联剂 KH-570 和季铵盐 QAS 对 HAP 陶瓷粉体进行改性, 获得 mHAP 粉体, 再利 用 SL技术打印 mHAP 浆料制得参与咬合动作的牙合 垫模型, 其中, mHAP 的使用在改善浆料流变性能 的同时, 还提升了陶瓷的抗菌活性。LIAN 等 ${ }^{[68]}$ 配制 出固含量为 $40 \%$ (体积分数)的 $\mathrm{ZrO}_{2}$ 水基陶瓷浆料, 并利用 SL 技术打印出了如图 4c 所示的 $\mathrm{ZrO}_{2}$ 陶瓷牙 桥, 烧结体的相对密度为 $98.58 \%$, 维氏硬度达 1398 $\mathrm{HV}$, 性能优于机加工得到的 $\mathrm{ZrO}_{2}$ 陶瓷牙桥。

此外, 电功能陶瓷元器件的小型化、精密化制 造需求拓展了陶瓷 SL 技术的应用领域。 CARPENTIER 等 ${ }^{[69]}$ 提出了基于 SL 技术的六极 $\mathrm{Ku}$ 带 $\mathrm{Ba}\left(\mathrm{Mg}_{1 / 3} \mathrm{Ta}_{2 / 3}\right) \mathrm{O}_{3}(\mathrm{BMT})$ 陶瓷滤波器的新型制造方
法, 其使用 BMT 陶瓷浆料打印得到的谐振器零件 可与银质谐振腔直接配合, 而不需要额外的定位装 置。制得的陶瓷滤波器中心工作频率为 $11.7 \mathrm{GHz}$, 带宽为 $200 \mathrm{MHz}$, 插入损耗为 $-0.3 \mathrm{~dB}$, 与带银质谐 振腔的常规六极准椭圆 TE113 滤波器相比体积减少 $41 \%$ 以, 可见 SL 技术在滤波器小型化制造方面的 优势, 有望推进电路的集成化发展进程。LI 等 ${ }^{[70]}$ 利用 SL 技术打印了如图 4d 所示的陶瓷谐振腔, 在 烧结体表面镀上镍和铜后, 经螺母、螺丝组装后无 需手动调谐, 节省了调试阶段所花费的人力和时间 成本。CHEN 等 ${ }^{[71]}$ 设计并利用 SL 技术制备了如图 $4 \mathrm{e}$ 所示的 $8 \times 8$ 二维 PZT 陶瓷超声传感器阵列, 相 对介电常数可达 $1390 \mathrm{pC} / \mathrm{N}$ 。在后续的排胶、烧结 过程中, 通过测定长宽高三个方向的收缩率, 供零 件尺寸设计时设置预补偿量, 从而确保烧结体具有 理想的外形尺寸和压电性能。WANG 等 ${ }^{[72]}$ 利用 BYK 对 $\mathrm{BaTiO}_{3}$ 陶瓷粉体进行化学改性, 制备出的浆料固 含量达到 $40 \%$ (质量分数)且具有良好的流动性, 之 后利用 SL 技术打印出了如图 4f 所示的压电传感器 素坏。制备的 $\mathrm{BaTiO}_{3}$ 陶瓷的压电常数为 $163 \mathrm{pC} / \mathrm{N}$, 相对介电常数为 2762 。LOU 等 ${ }^{[73]}$ 利用 SL 技术制备 $(1-x) \mathrm{MgTiO}_{3}-x \mathrm{CaTiO}_{3}$ 微波介质陶瓷, 并研究了 $\mathrm{CaTiO}_{3}$ 的含量对烧结体 $Q \times f$ 值的影响, 发现随着 $x$ 的增大, 虽然烧结体的晶粒尺寸分布相较于传统干 压成型获得的陶瓷更加均匀, 且 $Q \times f$ 值提高了 $26 \%$ 以上, 但同时也导致了浆料固化深度减小, 当固 
化深度小于 $1 \mathrm{~mm}$ 时素坏会产生层间位移, 从而 使烧结体的 Q×f 值降低。最终得到直径 $70 \mathrm{~mm}$ 、

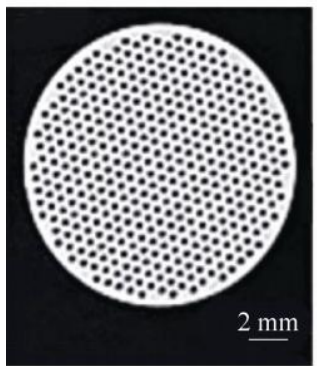

(a) HAP 多孔植入体 ${ }^{[65]}$

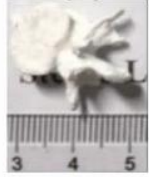

(b) $\beta$-TCP 脊椎骨模型 ${ }^{[66]}$

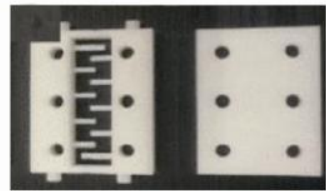

(d) 陶瓷谐振腔 [70]

厚度 $8 \mathrm{~mm}$ 的龙勃透镜, 体现了 SL 技术制备复杂结 构微波无源器件的优势。

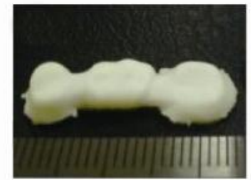

(c) $\mathrm{ZrO}_{2}$ 牙桥 ${ }^{[68]}$

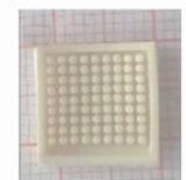

(e) PZT 超声传感器 [71]

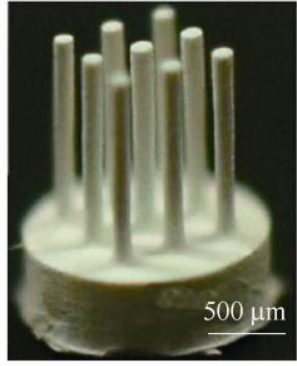

(f) $\mathrm{BaTiO}_{3}$ 压电传感器素坏 [72]

图 4 基于 SL 的功能陶瓷零件

通过前驱体制备陶瓷由于可从分子尺度设 计、制备温度低、陶瓷产率高、热稳定性好等优 点, 受到研究者广泛关注。近年来, 结合 SL 技 术的前驱体转化法为一些难以直接光固化的陶瓷 的成形提供了新的思路。JANA 等 ${ }^{[74]}$ 利用 SL 技 术制备具有蜂窝结构的陶瓷前驱体聚合物, 并将 浸渍有聚硅氮烷的陶瓷晶格在惰性气氛中经 1 $000{ }^{\circ} \mathrm{C}$ 高温热解转化为结构复杂且精度较高的 $\mathrm{SiCN}$ 陶瓷晶格。热解过程中前驱体有一定的质量 损失和体积收缩, 但浸渍后的前驱体在热解过程 中并未出现明显变形, 最后成功制备了孔隙率约 为 $93 \%$ 的多孔陶瓷。ECKEL 等 ${ }^{[75]}$ 分析了晶格结 构对陶瓷力学性能的影响, 其向树脂中加入硅氧 烷, 得到化学组成为 $\mathrm{SiO}_{1.34} \mathrm{C}_{1.25} \mathrm{~S}_{0.15}$ 的前驱体聚 合物, 并利用 SL 技术获得了图 $5 \mathrm{a} \sim 5 \mathrm{e}$ 所示的复 杂陶瓷零件。力学性能测试表明, 在同等的相对 密度下, 与通过传统成形方法得到的多孔陶瓷相 比, 该工艺制得的陶瓷零件具有更高的抗压强度 和抗弯强度, 密度为 $0.32 \mathrm{~g} / \mathrm{cm}^{3}$ 的多孔陶瓷的抗压 强度为 $47 \mathrm{MPa}$ 。在热解过程中, 前驱体聚合物收 缩均匀, 热解后获得耐热性好、孔隙和裂纹等微
观缺陷少的致密陶瓷零件。另外, 也有研究将 SL 与反应熔渗相结合, 制造 $\mathrm{SiC}$ 及其复合材料。TIAN 等 ${ }^{[76]}$ 对以酚醛树脂等为碳源前驱体的复合 $\mathrm{SiC}$ 陶 瓷零件的制造工艺进行了改进, 并开发了一种热 解后碳产率更高的新型光固化树脂, 其中造孔剂 为三甘醇, 光引发剂为安息香二甲基醚。研究发 现, 增加造孔剂的含量虽然可提高热解后的碳产 率, 但会减小固化深度, 最终确定合适的浆料组 分并获得了孔隙率为 $27 \%$ 、抗弯强度为 $4.48 \mathrm{MPa}$ 的碳预制体, 后在 $2300{ }^{\circ} \mathrm{C}$ 下进行熔融硅渗透, 使碳预制坏转化为 $\mathrm{SiC}$ 。XRD 结果显示, 在得到 的 $\mathrm{SiC}$ 样品中无残留的碳和硅。 $\mathrm{LU}$ 等 ${ }^{[77]}$ 以酚醛树 脂为碳源前驱体, 提出了一种 “立体光固化一凝胶 注模一反应溶渗” 混合技术, 研究了碳纤维含量对 $\mathrm{SiC}$ 陶瓷基复合材料涡轮叶片机械性能的影响, 发 现随着碳纤维含量的增加, $\mathrm{SiC}$ 陶瓷基复合材料浴 轮叶片的抗弯强度和断裂韧性均有所提高, 含有 $10 \% \sim 15 \%$ (质量分数) 碳纤维的样品抗弯强度和断 裂韧性分别可达 $350 \mathrm{MPa}$ 和 $4.49 \mathrm{MPa} \cdot \mathrm{m}^{1 / 2}$, 最终 成功制备了高性能 $\mathrm{SiC}$ 陶瓷基复合材料陶瓷空心 浴轮叶片。

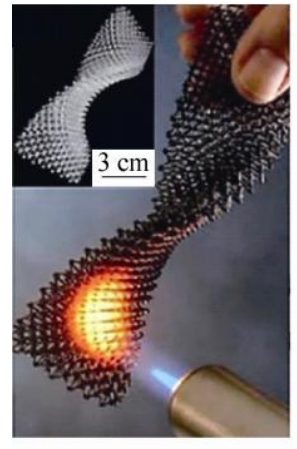

(a) $\mathrm{SiOC}$ 陶瓷

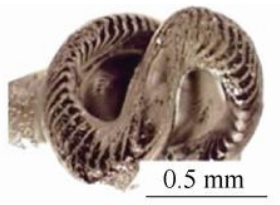

(b) 螺旋结构

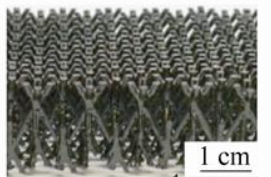

(d) 微点阵结构

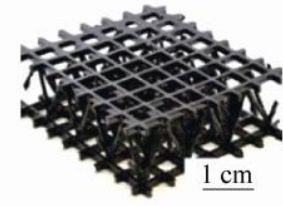

(c) 微点阵结构

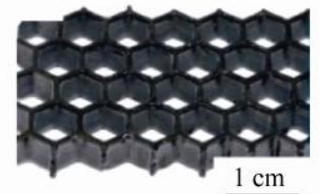

(e) 蜂窝结构

图 5 基于 SL 的前驱体转化陶瓷零件 ${ }^{[75]}$ 
近年来, 陶瓷 SL 技术已在电子通讯、生物医 疗等领域取得应用, 并有望应用于航空航天高端零 部件的制造。相比于传统陶瓷成形技术, SL 技术需 要复杂、高成本的光学系统, 且成形件的质量受激 光功率、扫描路径等多种因素的影响。此外, 陶瓷 SL 技术对材料的性能要求高, 目前对于许多常见的 氧化物陶瓷材料的制备已经有了较为深入的研究, 但仍少见有关 $\mathrm{Si}_{3} \mathrm{~N}_{4} 、 \mathrm{SiC}$ 等非氧化物陶瓷利用 $\mathrm{SL}$ 技术成形的案例。为拓展材料的应用范围、获得性 能理想的陶瓷零件, 还需要在原料的处理、新型浆 料的开发、扫描路径的优化等方面做进一步研究。

\section{2 数字光处理技术的应用}

由于 DLP 技术受限于光源和投影设备, 其最大 成形尺寸较小, 因而适合制造精度要求高、尺寸不 大的陶瓷零件, 尤其是在实现具有复杂多孔结构陶 瓷的成形方面具有明显优势。

DLP 技术在结构陶瓷领域应用广泛，且主要集 中在氧化物陶瓷上。 $\mathrm{Al}_{2} \mathrm{O}_{3} 、 \mathrm{ZrO}_{2}$ 等氧化物陶瓷因浆 料光固化难度相对较低、应用范围广而被作为陶瓷 DLP 打印最为主流的原料。HATZENBICHLER 等 ${ }^{[78]}$ 利用 DLP 技术制备出了 $\mathrm{Al}_{2} \mathrm{O}_{3}$ 陶瓷素坏, 烧结体的 抗弯强度可达 $510 \mathrm{MPa}$, 致密度达到 $99.6 \%$ 。基于 DLP 技术的基本原理, 奥地利的 Lithoz 公司成功开 发出用于商业的陶瓷光固化设备, 并利用树脂基
$\mathrm{Al}_{2} \mathrm{O}_{3}$ 陶瓷浆料为原料, 最终制备出了如图 6a 所示 的具有精密、复杂结构的 $\mathrm{Al}_{2} \mathrm{O}_{3}$ 陶瓷样件, 其抗弯 强度和相对密度分别达到 $427 \mathrm{MPa}$ 和 $99.3 \%$ 。 BORLAF 等 ${ }^{[80]}$ 使用 MelPers 4350 对 $\mathrm{ZrO}_{2}$ 粉体进行 表面改性, 并将改性后的粉末分散在丙烯酸酯中, 浆料通过 DLP 技术成形后得到如图 $6 \mathrm{~b}$ 所示的 $\mathrm{ZrO}_{2}$ 陶瓷螺钉, 烧结后的抗弯强度为 $741 \mathrm{MPa} 。 \mathrm{HE}$ 等 ${ }^{[2]}$ 利用 DLP 技术制备了带有凹槽的蜂窝状和三角状 $\mathrm{ZrO}_{2}$ 陶瓷, 烧结体由致密堆积的亚微米级晶粒组 成, 相对密度为 $97.14 \%$, 并且发生明显的收缩, 最大收缩率为 $35.26 \%$, 烧结后的陶瓷刀具形状和 尺寸如图 6c 所示。

与 SL 技术相同, 使用 DLP 技术制备非氧化 物陶瓷的案例也较少。HUANG 等 ${ }^{[82]}$ 通过表面氧化 法对 $\mathrm{Si}_{3} \mathrm{~N}_{4}$ 粉末进行改性, 提高了浆料的固化深度, 并成功地利用 DLP 技术打印出了一些复杂陶瓷零 件, 如图 6d 所示。严鹏飞等 ${ }^{[83]}$ 通过陶瓷前驱体转 化法预制 $\mathrm{Si}_{3} \mathrm{~N}_{4}$ 粉末, 并制备了低黏度浆料, 打印 出的陶瓷抗弯强度为 $308.5 \sim 333.2 \mathrm{MPa}$, 力学性能 优于通过传统方法经常压烧结得到的 $\mathrm{Si}_{3} \mathrm{~N}_{4}$ 陶瓷, 但其使用的浆料固含量过低, 样件水平方向的收 缩率达到了 $65 \%$ 以上。另外, Lithoz 公司利用 LCM 技术成功制造了如图 6e 所示的 $\beta$-SiAlON 陶瓷, 但未见其公开具体的技术细节 ${ }^{[84]}$ 。
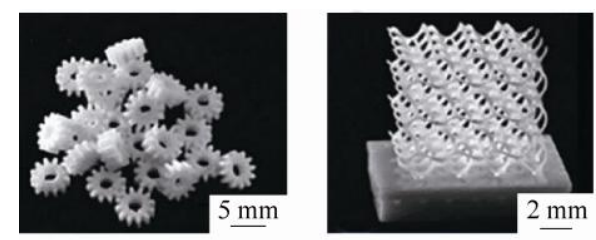

(a) Lithoz 公司制备的 $\mathrm{Al}_{2} \mathrm{O}_{3}$ 试样 [79]
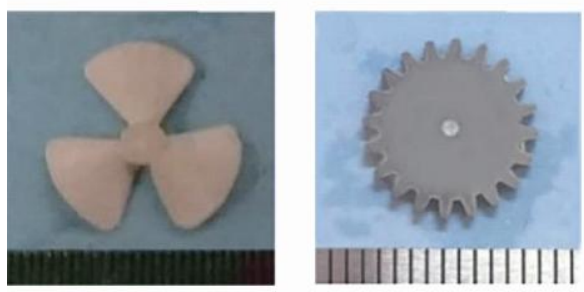

(d) 复杂形状的 $\mathrm{Si}_{3} \mathrm{~N}_{4}$ 陶瓷 [82]

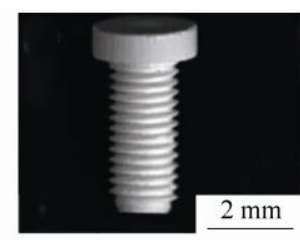

(b) $\mathrm{ZrO}_{2}$ 陶瓷螺钉 [80]

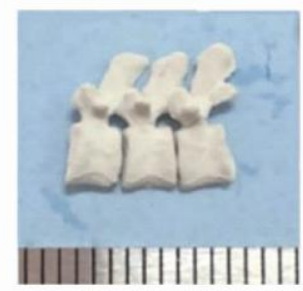

(e) $\beta$-SiAlON 叶轮 ${ }^{[84]}$

图 6 基于 DLP 的结构陶瓷零件

在功能陶瓷方面, DLP 技术目前主要被用于牙 齿、骨骼等生物陶瓷的制备。杨飞等 ${ }^{[85]}$ 研制了陶瓷 面曝光快速成形试验系统, 并使用自主开发的光固 化 TCP 陶瓷浆料对临界曝光强度、透射深度以及曝 光时间等工艺参数进行了优化, 最终打印出了如图
$7 \mathrm{a}$ 所示的多孔支架结构, 烧结后的致密件压缩强度 大于 $60 \mathrm{MPa}$, 可以满足作为骨组织支架的力学性能 要求。张航等 ${ }^{[86]}$ 分析了 $\beta-\mathrm{TCP}$ 光固化素坏与设计模 型之间的尺寸误差和素坏烧结过程中的收缩情况， 提出了烧结收缩补偿模型，在设计阶段对结构尺寸 
进行补偿, 从而在烧结收缩后得到期望尺寸的陶瓷 烧结体, 制备出了图 7b 所示的均匀多孔结构, 孔隙 率为 $56.42 \% \sim 64.79 \%$, 最大压缩强度为 $10.42 \sim$ $16.53 \mathrm{MPa}$, 其孔隙率和压缩强度均与人体松质骨接 近。除了合适的宏微观结构和力学性能之外, 良好 的生物性能也是生物陶瓷能够得到应用的关键性能 指标, 而通过 DLP 技术制备的陶瓷素坏在排胶过程 中有机物几乎可以完全去除, 故而保证了烧结体良 好的生物性能。CHEN 等 ${ }^{[87]}$ 综合研究了曝光时间和 浆料流变性能对固化深度的影响, 在单层曝光时间 $3 \mathrm{~s}$ 、浆料固含量 $42 \%$ (体积分数) 的条件下制备了如 图 7c 所示的 $\mathrm{ZrO}_{2}$ 全瓷牙, 相对密度达 $98.02 \%$, 维 氏硬度达 $12.62 \mathrm{GPa}$ 。经细胞毒性测试, 48 小时内 荧光标记的骨髓干细胞几乎全部分布在牙的外表 面, 说明烧结过程中有生物毒性的树脂等有机物成 分已被充分去除, 说明 DLP 技术在口腔修复领域具 有良好的应用前景。ZHANG 等 ${ }^{[88]}$ 利用 DLP 技术制 备 HAP 陶瓷, 发现通过向粉体中掺杂 $3 \%$ (质量分数) 的 $\mathrm{ZrO}_{2}$ 能够有效地减少烧结过程中 HAP 的相变分 解, 且掺杂后的陶瓷在力学性能得到明显改善的同时 也具有良好的生物相容性。焦晨 ${ }^{[89]}$ 使用自制的 DLP 设备打印了如图 7d 所示的三种 $\mathrm{ZrO}_{2}$ 陶瓷点阵结构, 对三种点阵结构进行压缩试验, 发现在相同条件下, 菱形十二面体结构具有最优的力学性能, 同时, 其 结构尺寸对压缩性能影响较小。细胞附着实验表明
制备的陶瓷具有良好的生物相容性。XU 等 ${ }^{[00]}$ 研制了 一种新型生物活性玻璃陶瓷(AP40mod), 利用 DLP 技术制备了具有理想力学性能的玻璃陶瓷支架, 并 在支架上接种了内皮祖细胞(EPC)和梅塞氏干细胞 (BMSC), 用于修复兔下领骨中的临界骨缺损。将支 架植入兔下骸骨缺损处, 经过一段时间的体内生长, 通过 X 射线观察到植入支架和骨组织之间的界限逐 渐模糊, 说明支架具有良好的组织相容性。同时, 通过 DLP 技术获得的支架抗弯强度高达 $52.7 \mathrm{MPa}$, 与 $3 \mathrm{DP}$ 技术打印的样品强度为 $17.5 \mathrm{MPa}$ 的结果相 比具有显著优势。

除生物陶瓷外，DLP 技术在电功能陶瓷的制备 上也取得了一些进展。TIAN 等 ${ }^{[91]}$ 采用 DLP 技术成 功制造了具有高孔隙率的 h- $\mathrm{BN}-\mathrm{SiO}_{2}$ 多孔陶瓷, 其 具有良好的力学性能和优异的介电性能, 在电磁波 传输领域具有潜在应用。XING 等 ${ }^{[92]}$ 将 DLP 技术用 于固体氧化物燃料电池电极的制备, 成功打印了致 密的氧化钎稳定氧化锆陶瓷, 在 $800{ }^{\circ} \mathrm{C}$ 的测试温度 下, 烧结后陶瓷的电导率为 $2.18 \times 10^{-2} \mathrm{~S} \cdot \mathrm{cm}^{-1}$, 性能 满足实际应用要求。CHABOK 等 ${ }^{[93]}$ 在成形基板上涂 覆聚二甲基硅氧烷(PDMS)薄膜, 使固化层易于剥 离, 利用 DLP 技术打印了如图 7e 所示的超声传感 器阵列结构, 并通过向 PZT 陶瓷浆料中加入烧结助 剂, 提高了烧结体的相对密度, 从而使零件的压电 性能提高了 $40 \%$ ～ $80 \%$ 。

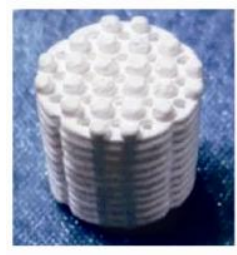

(a) $\beta$-TCP 多孔支架 ${ }^{[85]}$

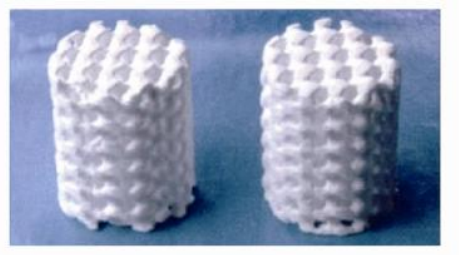

(b) $\beta$-TCP 多孔支架 $[86]$

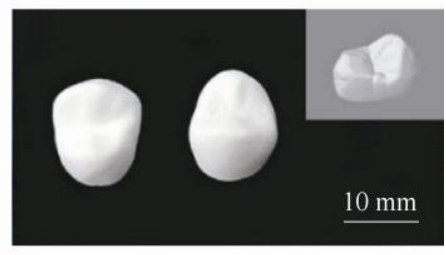

(c) $\mathrm{ZrO}_{2}$ 全瓷牙 [87]

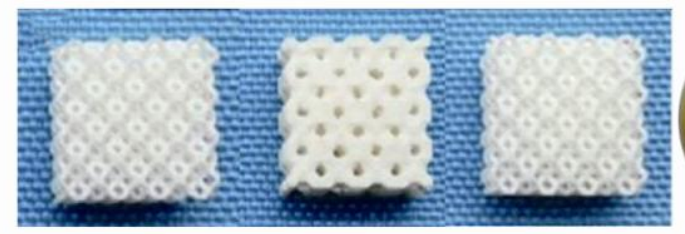

(d) $\mathrm{ZrO}_{2}$ 点阵结构 ${ }^{[89]}$

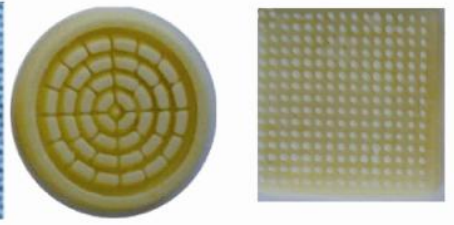

(e) PZT 压电陶瓷阵列 [93]

图 7 基于 DLP 的功能陶瓷零件

结合 DLP 技术的前驱体转化法制备复杂结构 陶瓷方面也有一些研究和应用。LI 等 ${ }^{[94]}$ 提出了采用 聚合物陶瓷前驱体、通过 DLP 技术制造具有复杂形 状的 SiBCN 陶瓷零件的方法, 其将聚嗍硅烷与丙烯 酸酯树脂单体混合均匀得到陶瓷前驱体, 优化配方
和打印参数, 得到高精度的前驱体原件, 热解前后 的外观及尺寸如图 8a 所示, 最终得到的 $\mathrm{SiBCN}$ 样 品收缩均匀、无明显变形, 该方法有望用于超音速 飞机机翼的热屏障层的制备。SCHMIDT 等 ${ }^{[95]}$ 将一 种液体光固化硅氧烷(TEGO RC 711)与两种高陶瓷 
产率的硅树脂混合, 然后利用 DLP 技术成形, 再经 过高温热解获得如图 $8 \mathrm{~b}$ 所示的结构致密、组分均匀 的 $\mathrm{SiO}_{2}$ 陶瓷。CHEN 等 ${ }^{[96]}$ 制备出了一种新型的 $\mathrm{SiC}$ 前驱体一一液体超支化聚碳硅烷(LHBPCS), 该前驱 体聚合物具有较高的光敏性和陶瓷产量 $\left(1000{ }^{\circ} \mathrm{C}\right.$ 时 陶瓷产量为 74.4\%), 利用 DLP 技术可以获得如图 $8 \mathrm{c}$ 所示无宏观缺陷的前驱体, 相较于其他前驱体,
通过 LHBPCS 获得的陶瓷收缩率明显较低。HE 等 ${ }^{[98]}$ 利用北京十维科技研发的 AUTOCERA 陶瓷 DLP 设 备打印出 $\mathrm{SiC}$ 陶瓷素坏, 随后进行高温排胶获得了 多孔 $\mathrm{SiC}$ 预制体, 再通过反复多次的聚碳硅烷前驱 体溶液浸渍和热解以提高陶瓷烧结体的密度和强 度。最后, 得到了如图 $8 \mathrm{~d}$ 所示的高精度、高质量的 复杂结构 $\mathrm{SiC}$ 陶瓷零件。

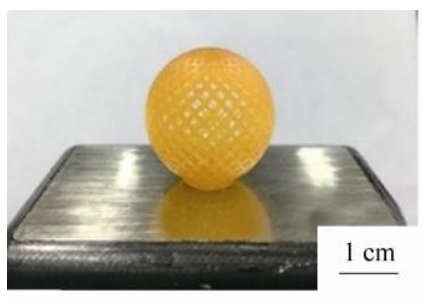

(a) SiBCN 陶瓷 [94]

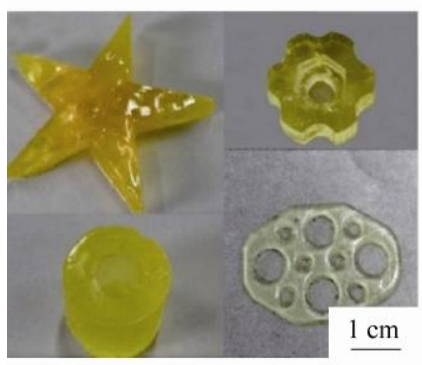

(c) $\mathrm{SiC}$ 陶瓷 [96]
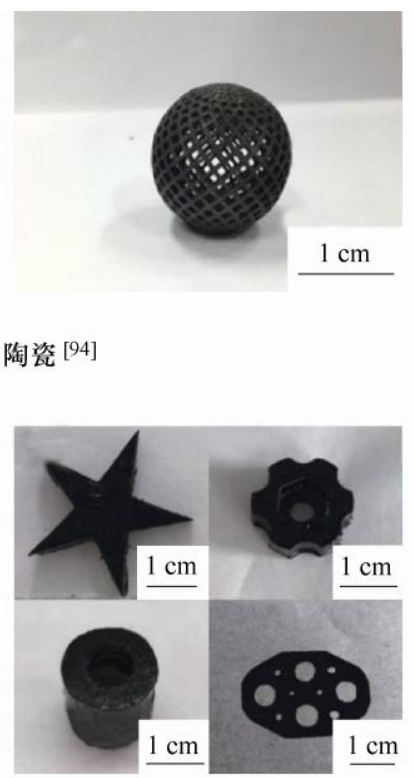

(b) $\mathrm{SiO}_{2}$ 陶瓷 $[95]$

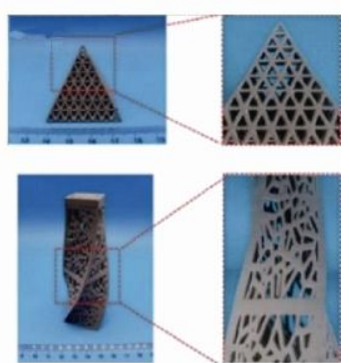

(d) $\mathrm{SiC}$ 陶瓷 [97]

图 8 基于 DLP 的前驱体转化陶瓷零件

目前，DLP 技术在陶瓷的光固化制造中的应 用大多集中在生物医疗等领域, 且使用的材料以 氧化物陶瓷为主。DLP 设备的离型膜、成形基板、 投影系统的性能等都对陶瓷的光固化成形起着关 键作用, 同时也限制了可用材料的范围, 更高性 能的组件以及新型的投影方式有待进一步研发。 总的来说, DLP 技术一改 SL 技术的点曝光的方 式, 大大提高了成形速度, 且自下而上的投影方 式避免了刮刀运动对固化层的影响, 一定程度上 提高了素坏的结构完整性, 故其应用和发展态势 良好, 但因此产生的内应力等问题不可忽略, 仍 然需要结合多学科的知识对相关设备、原料、成 形机理等进行深入研究。

\section{3 结论与展望}

陶瓷光固化技术因具有较高的成形精度和 质量而备受青睐。其中, SL 技术采用逐点扫描 的方式固化陶瓷浆料或膏体, 通过合理控制光斑 的形状和大小, 可以使成形精度和速度得到有效
提高, 但存在着浆料涂覆质量难以控制、刮刀运 动降低素坏的结构完整性等问题。DLP 技术采用 DMD 芯片进行面曝光, 同时速度显著提高, 且 衍生出了多种新颖的面曝光增材制造技术，但曝 光不均匀、固化层与离型膜分离拉力大等问题还 有待解决。陶瓷光固化技术的分辨率一般可以达 到 30 40 $\mu \mathrm{m}$, 且随着成形幅面的增大分辨率会 有所降低。同时, 一般要求所用浆料的固含量至 少为 $40 \%$ (体积分数) 以降低烧结收缩率, 烧结收 缩导致的误差可以通过预设尺寸补偿量得到很 大程度地消除。由于光固化成形无需模具, 对尺 寸和工艺参数进行迭代优化的周期较短, 因此可 以快速获得高精度的陶瓷零件。值得注意的是, 目前通过光固化技术制备的陶瓷素坏经烧结后 相对密度一般可达 97\% 99\%, 且部分已经可以 具备与传统方法所得陶瓷相当的力学性能, 而前 者可以实现更高的设计自由度。然而, 陶瓷光固 化技术本身的局限性也不容忽视。一方面, 陶瓷 光固化技术一般都需要对模型添加支撑以防止 塌陷变形, 但后续去支撑的步骤复杂、难以实现 
自动化, 正因如此, 陶瓷光固化工艺的生产流程 仍然不够规范, 且手动去支撑往往会降低零件的 结构完整性。另一方面, 由于光固化用陶瓷浆料 要求对紫外光具有良好的光敏性, 因此对紫外光 有较强吸收和散射作用的陶瓷材料难以通过光 固化成形, 为陶瓷光固化技术在某些领域的应用 造成阻碍。

国内外研究者针对上述两种陶瓷光固化技 术, 在开发新型陶瓷浆料、优化光固化工艺方案、 研发高性能陶瓷光固化设备等方面已取得一些进 展和突破, 并在精密铸造、生物医疗、航空航天、 文创设计等领域实现了一定的应用。但是, 陶瓷 光固化技术仍然有着较大的改进空间, 并且预计 在未来一段时间里将主要围绕以下几个方面 发展。

（1）适用原料种类的拓展: 目前, 陶瓷光固化 技术多用于 $\mathrm{Al}_{2} \mathrm{O}_{3} 、 \mathrm{ZrO}_{2}$ 等氧化物陶瓷的增材制 造, 而对于非氧化物陶瓷的研究较少。一些非氧 化物陶瓷如 $\mathrm{Si}_{3} \mathrm{~N}_{4} 、 \mathrm{SiC}$ 等具有优异的性能, 随着 航空航天等领域对高性能复杂结构陶瓷的精密化 制造的需求越来越大, 光固化技术应用于非氧化 物陶瓷的优势也日益凸显。另外, 相较于目前普 遍使用的树脂基陶瓷浆料, 水基陶瓷浆料具有黏 度低、污染小等优点, 且有利于实现更高的固含 量, 但利用水基陶瓷浆料光固化成形的素坯强度 较低, 其排胶、烧结工艺也尚不完善, 还有待深 入研究。

（2）梯度功能陶瓷的制备: 由于实际工作环境 的复杂性, 单一材料的陶瓷零件, 其性能往往难 以达到航空航天等极端条件下的应用标准。近年 来, 多种材料复合、结构和组分根据性能需求连 续变化的梯度功能陶瓷的制备成为材料领域的研 究热点。结合计算机辅助设计, 陶瓷光固化技术 在实现陶瓷结构的梯度变化上具有无可比拟的优 势, 目前, 也可以通过切换盛有不同浆料的料槽 来实现简单的层状梯度陶瓷的制备, 但是, 就现 有的技术而言, 曲面梯度陶瓷零件的制造仍然是 一个难题。未来的研究中, 如何实现陶瓷材料和 结构梯度的可控变化、最终制备出结构功能一体 化的梯度功能陶瓷, 将是陶瓷光固化技术的发展 方向之一。

（3）大尺寸陶瓷零件的制造：受到光学系统的 限制, 光固化成形台面尺寸较小, 难以制造大尺寸 陶瓷零件, 严重阻碍了陶瓷光固化领域的研究成果 向实际应用转化的进程, 因此, 大尺寸陶瓷零件的
光固化制造成为了一个亟待解决的难题。但是, 如 果只是简单地在增大成形面的同时增加投影光源的 数量, 则无疑会造成光固化设备使用、维护成本的 大幅提高。目前, 也有一些研究通过拼接式的曝光 以间接提高曝光面积, 但也存在着诸如加工误差 大、设备稳定性低等问题。另外，上述两种方法 得到的陶瓷素坏在拼接处都会产生局部过固化现 象, 导致最终得到的零件性能和使用寿命大大下 降。因此, 大成形台面、高精度、高稳定性的陶 瓷光固化设备及其配套的切片和控制等软件都有 待研发。

(4) 光固化过程缺陷的预测和监测: 光固化成 形的过程中存在着光敏树脂固化收缩、曝光能量不 均匀等复杂因素, 陶瓷素坯的成形过程会伴随着内 应力的积累, 容易产生开裂、变形等缺陷, 严重影 响陶瓷产品的成品率, 从而造成大量经济和时间成 本的耗费，而仅仅通过试错法预设固定的打印参数 并非高效的解决措施。因此, 需要对可能产生的缺 陷进行分析预测, 并根据实际情况动态地调整工艺 参数, 相关的数值模拟软件和控制软硬件的开发工 作有待加强。

（5）其他方面：目前，陶瓷光固化领域的研究 工作大多集中在材料和工艺上，对于新型光固化 理论和方法还需要进行深入系统地研究, 从而促 使更加高效、实用的陶瓷光固化技术的提出。同 时，有关的国家标准、行业标准、团体标准等体 系也需要不断完善, 进一步规范陶瓷光固化的原 料和工艺, 以保证产品质量满足特定应用环境的 要求。相关的科研和技术人才的培养工作也应该 得到加强和重视, 以更好地迎合行业发展对高端 复合人才的需求。另外, 陶瓷零件因其优异的性 能在各行各业都有着广阔的应用前景, 光固化技 术解决了高性能复杂陶瓷的制造难题, 有望拓展 到更多领域实现应用。

未来，随着陶瓷光固化的原料、设备、软件、 工艺、相关理论及标准等的不断完善和发展, 并结 合移动互联网、大数据等新兴技术，陶瓷光固化技 术必将朝着更加自动化、信息化、智能化、产业化 的方向迈进，有望推动国家制造业创新能力的提升、 信息化与工业化的深度融合以及重点领域关键问题 的突破, 并在航空航天、生物医疗等更多的领域发 挥不可或缺的积极作用, 为 “中国制造 2025 ” 战略 背景下我国从制造大国向制造强国的转变提供有力 支撑。 


\section{参 考 文 献}

[1] CHEN Zhangwei, LI Ziyong, LI Junjie, et al. 3D printing of ceramics: A review $[\mathrm{J}]$. Journal of the European Ceramic Society, 2019, 39(4): 661-687.

[2] ELORZ J A P-S, GONZáLEZ D F, VERDEJA L F. Structural materials : Ceramics[M]. Berlin : Springer International Publishing, 2019.

[3] MALIK H, DARWOOD A R J, SHAUNAK S, et al. Three-dimensional printing in surgery: A review of current surgical applications[J]. Journal of Surgical Research, 2015, 199(2): 512-522.

[4] JAVAID M, HALEEM A. 3D printed tissue and organ using additive manufacturing: An overview[J]. Clinical Epidemiology and Global Health, 2020, 8(2): 586-594.

[5] KO M, SHIN D, AHN H, et al. Informed ceramics: Multi-axis clay 3D printing on freeform molds[M]. Berlin: Springer International Publishing， 2018.

[6] 吴甲民, 陈敬炎, 陈安南, 等. 陶瓷零件增材制造技术 及在航空航天领域的潜在应用 [J]. 航空制造技术, 2017, 60(10): 40-49.

WU Jiamin, CHEN Jingyan, CHEN Annan, et al. Additive manufacturing of ceramic components and its potential application in aerospace field[J]. Aeronautical Manufacturing Technology, 2017, 60(10): 40-49.

[7] YAO Dongxu, XIA Yongfeng, ZENG Yuping, et al. Porous Si3N4 ceramics prepared via slip casting of Si and reaction bonded silicon nitride[J]. Ceramics International, 2011, 37(8): 3071-3076.

[8] LU Yuju, LIU Jingjing, REN Bo, et al. Room-temperature gelcasting of alumina with tartaric acid and glutaraldehyde[J]. Ceramics International, 2020, 46(8, Part A): 11432-11435.

[9] WU Jjiamin, LIU Shanshan, LIU Mengyue, et al. Preparation of dense $0.9 \mathrm{Al}_{2} \mathrm{O}_{3}-0.1 \mathrm{TiO}_{2}$ ceramics with highly improved microwave dielectric properties by a noncontaminated DCC method[J]. Ceramics International, 2018, 44(18): 22205-22211.

[10] RAHAMAN M N. Ceramic processing and sintering[M]. Boca Raton: CRC Press, 1995.

[11] 王志永, 赵宇辉, 赵吉宾, 等. 陶瓷增材制造的研究现 状与发展趋势 $[J]$. 真空, 2020，57(01)：67-75.

WANG Zhiyong, ZHAO Yuhui, ZHAO Jibin, et al. Research status and development trend of ceramic
precursors[J]. Vacuum, 2020, 57(01): 67-75.

[12] HWA L C, RAJOO S, NOOR A M, et al. Recent advances in $3 \mathrm{D}$ printing of porous ceramics: A review[J]. Current Opinion in Solid State and Materials Science, 2017, 21(6): 323-347.

[13] CHEN Annan, GAO Feng, LI Meng, et al. Mullite ceramic foams with controlled pore structures and low thermal conductivity prepared by SLS using core-shell structured polyamide12/FAHSs composites[J]. Ceramics International, 2019, 45(12): 15538-15546.

[14] 刘伟, 李能, 周标, 等. 复杂结构与高性能材料增材 制造技术进展 $[\mathrm{J}]$. 机械工程学报，2019，55(20)： 128-151, 159.

LIU Wei, LI Neng, ZHOU Biao, et al. Progress in additive manufacturing on complex structures and high-performance materials[J]. Journal of Mechanical Engineering, 2019, 55(20): 128-151, 159.

[15] 陈双，吴甲民，史玉升. 3D 打印材料及其应用概述 [J] 物理, 2018, 47(11): 715-724.

CHEN Shuang, WU Jiamin, SHI Yusheng. General introduction of 3D printing materials and their applications[J]. Physics, 2018, 47(11): 715-724.

[16] CHEN Annan, WU Jiamin, LIU Kai , et al. High-performance ceramic parts with complex shape prepared by selective laser sintering: A review[J]. Advances in Applied Ceramics, 2017, 117(4): 1-8.

[17] BEKAS D G, HOU Y, LIU Y, et al. 3D printing to enable multifunctionality in polymer-based composites : A review[J]. Composites Part B: Engineering, 2019, 179: 107540.

[18] 郭璐, 朱红. 陶瓷 3D 打印技术及材料的研究现状 [J]. 陶瓷学报，2020，41(1): 22-28.

GUO Lu, ZHU Hong. Progress in ceramic 3D printing technology and materials[J]. Journal of Ceramics, 2020, 41(1): 22-28.

[19] 吴甲民, 陈安南, 刘梦月, 等. 激光选区烧结用陶瓷材 料的制备及其成型技术 $[\mathrm{J}]$. 中国材料进展，2017， 36(Z1): 575-582.

WU Jiamin, CHEN Annan, LIU Mengyue, et al. Preparation of ceramic materials used for selective laser sintering and related forming methods[J]. Materials China, 2017, 36(Z1): 575-582.

[20] QUAN Haoyuan, ZHANG Ting, XU Hang, et al. 
Photo-curing 3D printing technique and its challenges[J].

Bioactive Materials，2020，5(1): 110-115.

[21] 黄丞俊, 伍海东, 黄容基, 等. 陶瓷增材制造(3D 打印) 技术研究进展[J]. 现代技术陶瓷, 2017, 38(4): 248-266. HUANG Miaojun, WU Haidong, HUANG Rongji, et al. A review on ceramic additive manufacturing (3D Printing)[J]. Advanced Ceramics, 2017, 38(4): 248-266.

[22] LEE K S, KIM R H, YANG D Y, et al. Advances in 3D nano/microfabrication using two-photon initiated polymerization[J]. Progress in Polymer Science, 2008, 33(6): 631-681.

[23] KAWATA S, SUN H B, TANAKA T, et al. Finer features for functional microdevices[J]. Nature, 2001, 412(6848): 697-698.

[24] HULL C W. Method and apparatus for production of three-dimensional objects by stereolithography: USA, EP0171069[P]. 1986-02-12.

[25] 潘翔, 莫健华, 冯昕, 等. 光固化成形中的变补偿量扫 描研究[J]. 激光杂志, 2007, 28(4): 75-76.

PAN Xiang, MO Jianhua, FENG Xin, et al. Research on scanning of variable radius compensation in SLA[J]. Laser Journal， 2007， 28(4): 75-76.

[26] 李子健. SLA 变光斑扫描方法研究与实现[D]. 武汉: 华 中科技大学, 2019 .

LI Zijian. Research and implementation of SLA variable spot scanning method[D]. Wuhan: Huazhong University of Science \& Technology, 2019.

[27] 胥光申, 赵万华, 卢秉恒. 用高分辨率快速成形系统制 作细微结构 [J]. 机械工程学报, 2005, 41(9): 82-85. XU Guangshen, ZHAO Wanhua, LU Bingheng. Fabrication of microstructures using high-resolution stereolithography system[J]. Journal of Mechanical Engineering, 2005, 41(9): 82-85.

[28] 胥光申, 邓攀, 赵万华, 等. 高分辨率激光快速成形系 统研究与开发 $[J]$. 机械工程学报, 2005, 41(2): 127-131. XU Guangshen, DENG Pan, ZHAO Wanhua, et al. Research and development of high-resolution stereolithography system for small objects[J]. Journal of Mechanical Engineering, 2005, 41(2): 127-131.

[29] GRIFFITH M L, HALLORAN J W. Freeform Fabrication of Ceramics via Stereolithography[J]. Journal of the American Ceramic Society, 1996, 79(10): 2601-2608.

[30] BÁRTOLO P J. Stereolithography: Materials, processes and applications[M]. Boston: Springer, 2011.

[31] DING Guojiao, HE Rujie, ZHANG Keqiang, et al. Dispersion and stability of $\mathrm{SiC}$ ceramic slurry for stereolithography[J]. Ceramics International, 2020, 46(4): 4720-4729.

[32] SUN Jinxing, BINNER J, BAI Jiaming. Effect of surface treatment on the dispersion of nano zirconia particles in non-aqueous suspensions for stereolithography[J]. Journal of the European Ceramic Society, 2019, 39(4) : 1660-1667.

[33] 阎相忠. 基于光固化水基陶瓷浆料的义齿成型研究 [D]. 兰州: 兰州理工大学, 2019 .

YAN Xiangzhong. The research on denture forming based on stereolithography for an aqueous-based ceramic suspension[D]. Lanzhou: Lanzhou University of Technology, 2019.

[34] 宁会峰, 阎相忠, 朱悦, 等. 水基光固化陶瓷浆料的黏 度与分散性研究 $[\mathrm{J}]$. 硅酸盐通报，2017，36(11)： 3944-3949.

NING Huifeng, YAN Xiangzhong, ZHU Yue, et al. Research on viscosity and dispersity of aqueous ceramic suspension for stereolithography $[\mathrm{J}]$. Bulletin of the Chinese Ceramic Society, 2017， 36(11): 3944-3949.

[35] WANG Zhen, HUANG Chuanzhen, WANG Jun, et al. Development of a novel aqueous hydroxyapatite suspension for stereolithography applied to bone tissue engineering[J]. Ceramics International, 2019, 45(3): 3902-3909.

[36] 刘丹丹, 李芳, 张小敏, 等. 光固化 3D 打印用于陶瓷 制备的研究进展 [J]. 杭州师范大学学报, 2019, 18(6): 576-580.

LIU Dandan, LI Fang, ZHANG Xiaomin, et al. Research progress in the ceramic production by stereolithography $3 \mathrm{D}$ printing[J]. Journal of Hangzhou Normal University, 2019, 18(6): 576-580

[37] CORCIONE C E, GRECO A, LICCIULLI A, et al. Ceramic components built by stereolithography[C]//AMST'02 Advanced Manufacturing Systems and Technology. Udine: International Centre for Mechanical Sciences, 2002, 731-739.

[38] SUN Cheng, ZHANG Xiang. The influences of the material properties on ceramic micro-stereolithography[J]. Sensors and Actuators A: Physical, 2002, 101(3): 364-370.

[39] BADEV A, ABOULIATIM Y, CHARTIER T, et al. 
Photopolymerization kinetics of a polyether acrylate in the presence of ceramic fillers used in stereolithography[J]. Journal of Photochemistry and Photobiology A: Chemistry, 2011, 222(1): 117-122.

[40] CHARTIER T, BADEV A, ABOULIATIM Y, et al. Stereolithography process: Influence of the rheology of silica suspensions and of the medium on polymerization kinetics - Cured depth and width[J]. Journal of the European Ceramic Society, 2012，32(8): 1625-1634.

[41] LIU Yao, CHENG Lijin, LI Hao, et al. Formation mechanism of stereolithography of $\mathrm{Si}_{3} \mathrm{~N}_{4}$ slurry using silane coupling agent as modifier and dispersant[J]. Ceramics International, 2020, 46(10, Part A): 14583-14590.

[42] LIU Xiaoyan, ZOU Bin, XING Hongyu, et al. The preparation of $\mathrm{ZrO}_{2}-\mathrm{Al}_{2} \mathrm{O}_{3}$ composite ceramic by SLA-3D printing and sintering processing[J]. Ceramics International, 2020, 46(1): 937-944.

[43] XING Hongyu, ZOU Bin, WANG Xinfeng, et al. Fabrication and characterization of $\mathrm{SiC}$ whiskers toughened $\mathrm{Al}_{2} \mathrm{O}_{3}$ paste for stereolithography 3D printing applications[J]. Journal of Alloys and Compounds, 2020, 828: 154347.

[44] TAKAGI T, NAKAJIMA N. Photoforming applied to fine machining $[\mathrm{C}] / /$ Micro Electro Mechanical Systems, Proceedings IEEE Micro Electro Mechanical Systems, February 10, 1993, Fort Lauderdale: Institute of Electrical and Electronics Engineers, 1993: 173-178.

[45] BERTSCH A, ZISSI S, JéZéQUEL J Y, et al. Microstereophotolithography using a liquid crystal display as dynamic mask-generator[J]. Microsystem Technologies, 1997, 3(2): 42-45.

[46] KANEKO Y, TAKAHASHI K. LADp-6 UV exposure system for photolithography and rapid prototyping using DMD projector[C]//SID Conference Record of the International Display Research Conference, Saitama Prefecture: ITE Technical, 2001, 42.

[47] TUMBLESTON J R, SHIRVANYANTS D, ERMOSHKIN $\mathrm{N}$, et al. Continuous liquid interface production of $3 \mathrm{D}$ objects[J]. Science，2015，347(6228): 1349-1352.

[48] KELLY B E, BHATTACHARYA I, HEIDARI H, et al. Volumetric additive manufacturing via tomographic reconstruction[J]. Science, 2019, 363(6431): 1075-1079.

[49] 邱志惠, 陈号, 黄祺, 等. 面曝光 3D 打印机光强检测
补偿系统研究与实现 $[\mathrm{J}]$. 西安交通大学学报, 2017, 51(08): 77-83.

QIU Zhihui, CHEN Hao, HUANG Qi, et al. Measurement and compensation of DLP projector light intensity for mask projection stereolithography[J]. School of Mechanical Engineering, Xi'an Jiaotong University, 2017, 51(08): 77-83.

[50] 冊立芳, 赵立东, 邱健康, 等. 面向面曝光 3D 打印的 模型自适应光照均匀化方法 [J]. 信号处理, 2017, 33(10): 1308-1316.

WU Lifang, ZHAO Lidong, QIU Jiankang, et al. The model adaptive energy homogenization scheme for mask projection 3D printing[J]. Faculty of Information Technology, 2017, 33(10): 1308-1316.

[51] 陈国大. 数字投影微光刻 $3 \mathrm{D}$ 打印关键技术研究[D]. 广 州: 华南理工大学, 2019.

CHEN Gouda. Research on key techniques of digital projection microlithographic 3D printing[D]. Guangzhou: South China University of Technology, 2019.

[52] EMIL J, OSCAR L M, JAN J, et al. Influence of resin composition on the defect formation in alumina manufactured by stereolithography[J]. Materials, 2017, 10(2): 138 .

[53] WU Chenming, YI Ran, LIU Yongjin, et al. Delta DLP $3 \mathrm{D}$ printing with large size[C]//2016 IEEE/RSJ International Conference on Intelligent Robots and Systems. Daejeon: IEEE, 2016: 2155-2160.

[54] 赵立东. 融合智能信息处理的面曝光 3D 打印关键技术 研究[D]. 北京: 北京工业大学, 2018.

ZHAO Lidong. Research on key issues of mask projection $3 \mathrm{D}$ priting with intelligent information processing[D]. Beijing: Beijing University of Technology, 2018.

[55] 权坤. 双紫外掩模面成型机理及成型精度研究[D]. 西 安: 西安科技大学, 2019 .

QUAN Kun. Research on forming mechanism and accuracy of double ultraviolet mask projection stereolithography technology[D]. Xi'an: Xi'an University of Science and Technology, 2019.

[56］陈凌香. 基于 DLP 技术的陶瓷骨支架 $3 \mathrm{D}$ 打印机的研制 [D]. 广州: 广东工业大学, 2019.

CHEN Lingxiao. Development of 3D printer based on DLP technology for ceramic bone support[D]. Guangzhou: Guangdong University of Technology, 2019. 
[57] XING Zhanwen, LIU Weiwei, CHEN Yao, et al. Effect of plasticizer on the fabrication and properties of alumina ceramic by stereolithography-based additive manufacturing $[\mathrm{J}]$. Ceramics International， 2018，44(16): 19939-19944.

[58] ZHOU Maopeng, LIU Wei, WU Haidong, et al. Preparation of a defect-free alumina cutting tool via additive manufacturing based on stereolithography Optimization of the drying and debinding processes[J]. Ceramics International, 2016，42(10): 11598-11602.

[59] ZHANG Xiang, JIANG Xiaoning, SUN Cheng. Micro-stereolithography of polymeric and ceramic microstructures[J]. Sensors \& Actuators: A Physical, 1999, 77(2): 149-156.

[60] HE Li, FEI Fan, WANG Wenbo, et al. Support-free ceramic stereolithography of complex overhanging structures based on an elasto-viscoplastic suspension feedstock[J]. ACS Applied Materials \& Interfaces, 2019, 11(20): 18849-18857.

[61] SONG S Y, PARK M S, LEE D, et al. Optimization and characterization of high-viscosity $\mathrm{ZrO}_{2}$ ceramic nanocomposite resins for supportless stereolithography[J]. Materials \& Design, 2019, 180: 107960.

[62] XING Hongyu, ZOU Bin, LIU Xiaoyan, et al. Effect of particle size distribution on the preparation of ZTA ceramic paste applying for stereolithography 3D printing[J]. Powder Technology, 2020，359: 314-322.

[63] BRINCKMANN S A, PATRA N, YAO J, et al. Stereolithography of SiOC polymer derived ceramics filled with $\mathrm{SiC}$ micronwhiskers[J]. Advanced Engineering Materials, 2018, 20(11): 1800593.

[64] LIU Yao, ZHAN Lina, HE Yu, et al. Stereolithographical fabrication of dense $\mathrm{Si}_{3} \mathrm{~N}_{4}$ ceramics by slurry optimization and pressure sintering $[\mathrm{J}]$. Ceramics International, 2020, 46(2): 2063-2071.

[65] CHEN Qinghua, ZOU Bin, LAI Qingguo, et al. A study on biosafety of HAP ceramic prepared by SLA-3D printing technology directly[J]. Journal of the Mechanical Behavior of Biomedical Materials, 2019, 98: 327-335.

[66] 贲玥. 3D 打印用 $\beta-\mathrm{TCP}$ 生物陶瓷粉体的制备及其性能 研究[D]. 徐州：江苏师范大学, 2018 .

BEN Yue. Fabrication and performance research of $\beta$-TCP bioceramic powders for 3D printing[D]. Xuzhou: Jiangsu Normal University, 2018.
[67] MAKVANDI P, CORCIONE C E, PALADINI F, et al. Antimicrobial modified hydroxyapatite composite dental bite by stereolithography[J]. Polymers for Advanced Technologies, 2018, 29(1): 364-371.

[68] LIAN Qin, SUI Wenquan, WU Xiangquan, et al. Additive manufacturing of $\mathrm{ZrO}_{2}$ ceramic dental bridges by stereolithography[J]. Rapid Prototyping Journal, 2018, 24(1): 114-119.

[69] CARPENTIER L, DELHOTE N, VERDEYME S, et al. Compact $\mathrm{Ku}$ band filter based on BMT dielectric resonators made in a single part using 3D ceramic stereolithography process $[\mathrm{C}] / / 2012$ IEEE/MTT-S International Microwave Symposium Digest. Montreal: Institute of Electrical and Electronics Engineers， 2012， 1-3.

[70] LI Jin, HUANG Guanlong, YUAN Tao, et al. A Ku-Band Wideband 3-D Printed Interdigital Bandpass Filter Free of Post Fabrication Tuning[C]//Proceedings of the 2018 IEEE International Symposium on Antennas and Propagation \& USNC/URSI National Radio Science Meeting, Boston: Institute of Electrical and Electronics Engineers, 2018, 1439-1440.

[71] CHEN Yan, BAO Xiulan, WONG Chi-Man, et al. PZT ceramics fabricated based on stereolithography for an ultrasound transducer array application[J]. Ceramics International, 2018, 44(18): 22725-22730.

[72] WANG Wei, SUN Jinxing, GUO Binbin, et al. Fabrication of piezoelectric nano-ceramics via stereolithography of low viscous and non-aqueous suspensions[J]. Journal of the European Ceramic Society, 2020, 40(3): $682-688$

[73] LOU Yihui, WANG Fei, LI Zijian, et al. Fabrication of high-performance $\mathrm{MgTiO}_{3}-\mathrm{CaTiO}_{3}$ microwave ceramics through a stereolithography-based $3 \mathrm{D}$ printing $[\mathrm{J}]$. Ceramics International, 2020, 46(10, Part B): 1697916986

[74] JANA P, SANTOLIQUIDO O, ORTONA A, et al. Polymer-derived SiCN cellular structures from replica of $3 \mathrm{D}$ printed lattices[J]. Journal of the American Ceramic Society, 2018, 101(7): 2732-2738

[75] ECKEL Z C, ZHOU Chaoyin, MARTIN J H, et al. Additive manufacturing of polymer-derived ceramics[J]. Science, 2016, 351(6268): 58-62.

[76] TIAN Xiaoyong, ZHANG Weigang, LI Dichen, et al. 
Reaction-bonded $\mathrm{SiC}$ derived from resin precursors by Stereolithography[J]. Ceramics International, 2011, 38(1): 589-597.

[77] LU Z L, LU F, CAO J W, et al. Manufacturing properties of turbine blades of carbon fiber-reinforced $\mathrm{SiC}$ composite based on stereolithography[J]. Materials and Manufacturing Processes, 2014, 29(2): 201-209.

[78] HATZENBICHLER M, GRUBER S, STAMPFL J, et al. DLP-based light engines for additive manufacturing of ceramic parts[R]. 8254-82540E-10. SPIE MOEMSMEMS. International Society for Optics and Photonics, 2012.

[79] SCHWENTENWEIN M, HOMA J. Additive manufacturing of dense alumina ceramics[J]. International Journal of Applied Ceramic Technology, 2015, 12(1): 1-7.

[80] BORLAF M, SERRA-CAPDEVILA A, COLOMINAS $\mathrm{C}$, et al. Development of UV-curable $\mathrm{ZrO}_{2}$ slurries for additive manufacturing (LCM-DLP) technology[J]. Journal of the European Ceramic Society, 2019，39(13): 3797-3803.

[81] HE Rongxuan, LIU Wei, WU Ziwei, et al. Fabrication of complex-shaped zirconia ceramic parts via a DLPstereolithography-based 3D printing method[J]. Ceramics International, 2018, 44(3): 3412-3416.

[82] HUANG Rongji, JIANG Qiangguo, WU Haidong, et al. Fabrication of complex shaped ceramic parts with surface-oxidized $\mathrm{Si}_{3} \mathrm{~N}_{4}$ powder via digital light processing based stereolithography method[J]. Ceramics International, 2019, 45(4): 5158-5162.

[83] 严鹏飞, 严彪, 王联风, 等. 氮化硅光固化增材制造工 艺与性能的研究 [J]. 有色金属材料与工程, 2018, 39(6): 12-15.

YAN Pengfei, YAN Biao, WANG Lianfeng, et al. Study on stereo lithography additive manufacturing process and properties silicon nitride[J]. Nonferrous Metal Materials and Engineering, 2018, 39(6): 12-15.

[84] https:// www. lithoz. com/produkte/material.

[85] 杨飞, 连芩, 武向权, 等. 陶瓷面曝光快速成型工艺研 究[J]. 机械工程学报, 2017, 53(7): 138-144.

YANG Fei, LIAN Qin, WU Xiangquan, et al. Ceramics fabrication using rapid prototyping of mask projection stereolithography[J]. Journal of Mechanical Engineering, 2017, 53(7): 138-144.
[86] 张航, 许宋锋, 熊胤泽, 等. 多孔 $\beta$-TCP 生物陶瓷 DLP 打印工艺研究[J]. 机械工程学报, 2019, 55(15): 81-87. ZHANG Hang, XU Songfeng, XIONG Yinze, et al. Fabrication of porous $\beta$-TCP bioceramics using digital light processing[J]. Journal of Mechanical Engineering, 2019, 55(15): 81-87.

[87] CHEN Fen, ZHU Hao, WU Jiamin, et al. Preparation and biological evaluation of $\mathrm{ZrO}_{2}$ all-ceramic teeth by DLP technology[J]. Ceramics International，2020，46(8, Part A): $11268-11274$.

[88] ZHANG Jiancheng, HUANG Da, LIU Shuifeng, et al. Zirconia toughened hydroxyapatite biocomposite formed by a DLP 3D printing process for potential bone tissue engineering $[\mathrm{J}]$. Materials Science and Engineering: C, 2019, 10: 110054

[89] 焦晨. DLP 光固化成形晶格单元结构氧化锆陶瓷性能 研究[D]. 南京: 南京航空航天大学, 2019.

JIAO Chen. Study on properties of lattice zirconia structures prepared via DLP method[D]. Nanjing: Nanjing University of Aeronautics and Astronautics, 2019.

[90] XU Fangfang, REN Hui, ZHENG Mingjie, et al. Development of biodegradable bioactive glass ceramics by DLP printed containing EPCs/BMSCs for bone tissue engineering of rabbit mandible defects[J]. Journal of the Mechanical Behavior of Biomedical Materials, 2020, 103: 103532.

[91] TIAN Zhuo, YANG Yuping, WANG Yong, et al. Fabrication and properties of a high porosity h-BN-SiO ceramics fabricated by stereolithography-based 3D printing[J]. Materials Letters, 2019, 236: 144-147.

[92] XING Bohang, CAO Chuanru, ZHAO Weiming, et al. Dense $8 \mathrm{~mol} \%$ yttria-stabilized zirconia electrolyte by DLP stereolithography[J]. Journal of the European Ceramic Society, 2020, 40(4): 1418-1423.

[93] CHABOK H, CHI Z, YONG C, et al. Ultrasound Transducer Array Fabrication Based on Additive Manufacturing of Piezocomposites[C]//ASME/ISCIE 2012 International Symposium on Flexible Automation. St. louis: American Society of Mechanical Engineers, 2012, 433-444.

[94] LI Shan, DUAN Wenyan, ZHAO Tong, et al. The fabrication of $\mathrm{SiBCN}$ ceramic components from preceramic polymers by digital light processing (DLP) 3D 
printing technology[J]. Journal of the European Ceramic Society, 2018, 38(14): 4597-4603.

[95] SCHMIDT J, COLOMBO P. Digital light processing of ceramic components from polysiloxanes[J]. Journal of the European Ceramic Society, 2018，38(1): 57-66.

[96] CHEN Jiangshan, WANG Yuanjie, PEI Xueliang, et al. Preparation and stereolithography of $\mathrm{SiC}$ ceramic precursor with high photosensitivity and ceramic yield[J]. Ceramics International, 2020，46(9): 13066-13072.
[97] HE Rujie, DING Guojiao, ZHANG Keqiang, et al. Fabrication of $\mathrm{SiC}$ ceramic architectures using stereolithography combined with precursor infiltration and pyrolysis[J]. Ceramics International, 2019，45(11): 14006-14014.

作者简介: 吴甲民(通信作者), 男, 1984 年出生, 博士, 副教授, 博士 研究生导师。主要研究方向为陶瓷增材制造技术及其应用。

E-mail: jiaminwu@hust.edu.cn 